Smooth, second order, non-negative meshfree approximants selected by maximum entropy, Cyron, ;Arroyo, M.; Ortiz, M.; International Journal for Numerical Methods in Engineering, Vol. 79, pp. 1605-1632, 2009

\title{
Smooth, second order, non-negative meshfree approximants selected by maximum entropy ${ }^{\ddagger}$
}

\author{
C. J. Cyron ${ }^{1}$, M. Arroyo ${ }^{2}$ and M. Ortiz ${ }^{3, *, \dagger}$ \\ ${ }^{1}$ Chair for Computational Mechanics, Technical University of Munich, Boltzmannstr. 15, \\ 85748 Garching, Germany \\ ${ }^{2}$ Department of Applied Mathematics 3, LaCàN, Universitat Politècnica de Catalunya (UPC), \\ Barcelona 08034, Spain \\ ${ }^{3}$ Graduate Aeronautical Laboratories, California Institute of Technology, Pasadena, CA 91125, U.S.A.
}

\begin{abstract}
SUMMARY
We present a family of approximation schemes, which we refer to as second-order maximum-entropy (max-ent) approximation schemes, that extends the first-order local max-ent approximation schemes to second-order consistency. This method retains the fundamental properties of first-order max-ent schemes, namely the shape functions are smooth, non-negative, and satisfy a weak Kronecker-delta property at the boundary. This last property makes the imposition of essential boundary conditions in the numerical solution of partial differential equations trivial. The evaluation of the shape functions is not explicit, but it is very efficient and robust. To our knowledge, the proposed method is the first higher-order scheme for function approximation from unstructured data in arbitrary dimensions with non-negative shape functions. As a consequence, the approximants exhibit variation diminishing properties, as well as an excellent behavior in structural vibrations problems as compared with the Lagrange finite elements, MLS-based meshfree methods and even B-Spline approximations, as shown through numerical experiments. When compared with usual MLS-based second-order meshfree methods, the shape functions presented here are much easier to integrate in a Galerkin approach, as illustrated by the standard benchmark problems.
\end{abstract}

KEY WORDS: meshfree methods; convex approximants; maximum entropy; B-Splines

\footnotetext{
*Correspondence to: M. Ortiz, Graduate Aeronautical Laboratories, California Institute of Technology, Pasadena, CA 91125, U.S.A.

†E-mail: ortiz@aero.caltech.edu

${ }^{\ddagger}$ This article was published online on 7 May 2009. An error was subsequently identified. This notice is included in the online and print versions to indicate that both have been corrected [2 June 2009].
} 


\section{J. CYRON, M. ARROYO AND M. ORTIZ}

\section{INTRODUCTION}

We present an extension of a recently proposed meshfree method, the local maximum-entropy (LME) approximation schemes [1], which contrary to most common approaches, notably the moving least-squares (MLS) method, is based on non-negative shape functions. This self-imposed constraint on the shape functions makes it less straightforward to develop higher-order methods, but endows the approximants with the mathematical structure of convex geometry, which results in a number of desirable features of the approximants. In [1], some properties of the so-called convex approximants (non-negative and reproducing exactly affine functions) were identified. In particular, such approximants satisfy a weak delta-Kronecker property from the outset, in sharp contrast with the MLS-based approximants used in popular meshfree methods such as EFG or RKPM [2,3]. Thus, the complications in implementing essential boundary conditions of standard approaches [4] are circumvented. The fact that B-Spline approximants are interpolatory at the ends of a patch is a particular instance of this property of convex approximants; on the other hand, it is well known [5] that local non-negative approximants exhibit variation diminishing properties, a feature of B-Splines and Non-uniform rational B-Spline (NURBS) approximants that has received much attention recently $[6,7]$.

In the present paper, we present a method to construct second-order maximum-entropy (SME) approximation schemes, circumventing the difficulties pointed out in [1]. The rationale behind the method is summarized next. Since the approximants are non-negative and form a partition of unity, at each point in space they can be interpreted as a discrete probability distribution. The definition of the approximants is then viewed as a statistical inference problem in which the shape functions (discrete probability) are the unknowns. Here, the first- and second-order reproducing conditions are viewed as constraints on the probability distribution, and the approximants are obtained as those that maximize the information entropy [8], i.e. the least biased choice consistent with what is known about the distribution (the consistency constraints) [9]. From this viewpoint, defining the approximants locally as polynomials or rational functions involves an arbitrary choice to be avoided. The resulting approximants retain the main features of the LME approximants, namely the smoothness $\left(C^{\infty}\right.$ if some easily achievable strict complementarity conditions are met), the dimension independence (a single routine with the space dimension as an argument computes the approximants in any spacial dimension), the weak delta-Kronecker property, and the efficient and robust evaluation of the shape functions through duality methods.

As compared with the approximants explicitly constructed in terms of polynomials, e.g. finite element spaces, B-Spline or NURBS, meshfree approximants require more calculations. MLS approximants require the solution of a linear system at each evaluation point, while for maximumentropy (max-ent) approximants a convex non-linear optimization problem must be solved. Furthermore, numerical quadrature is more expensive in meshfree methods, often relying on background cells. It is sometimes believed that the flexibility of meshfree methods does not outweigh these drawbacks. Nevertheless, we believe that the key feature of meshfree methods is the accuracy of the solutions they provide. When accuracy is taken into account, the additional cost of shape function evaluation and quadrature can become irrelevant, particularly in non-linear problems.

In some sense, max-ent approximants are meshfree relatives of B-Spline approximants. Indeed, in [1] it was rigorously shown that the Delaunay approximants are limits of the LME approximants when a locality parameter is drawn to infinity. Here we show that quadratic B-splines are closely related to SME approximants: both comply with a positivity requirement even in case of higher order approximation. Furthermore one can construct SME functions, which closely resemble second 
order B-splines. We show through numerical experiments that the extra smoothness of the SME approximants grants very accurate solutions as compared with B-Splines, while allowing for unstructured discretizations. In contrast to these non-negative approximants, MLS approximants are meshfree relatives of Lagrange interpolants in the sense that for second- and higher-order accuracy they necessarily produce shape functions with negative values. Even first-order MLS approximants with non-uniform node distributions or close to the boundary produce negative values of the shape functions. Also, in particular 1D cases the Lagrange approximants are recovered from MLS.

The outline of the paper is as follows. In Section 2, we introduce the general setup of convex approximants, the max-ent principle as a selection device, and review the first-order max-ent approximants. Section 3 highlights the feasibility issues when considering higher-order nonnegative approximants in 1D. A general method for ensuring the feasibility conditions is presented in $1 \mathrm{D}$ and the SME approximants defined. The $1 \mathrm{D}$ case serves as the building block for multiple dimensions, thanks to the special properties at the boundary of polyhedral domains of convex approximants, as discussed in Section 4. Section 5 collects numerical examples of structural vibrations and the Poisson equation in 2D and 3D, in which finite elements, the MLS, the max-ent, and B-Spline approximants are compared.

\section{SETUP}

Let us first recall the first-order max-ent approximants presented in [1]. See also [10,11] for the relation of the present formulation with the relative entropy maximization approach. Here, for the sake of completeness, we review some of the topics of particular relevance to the present work. See also [12] for related work.

Let $u: \Omega \subset \mathbb{R}^{d} \rightarrow \mathbb{R}$ be a function whose values $\left\{u_{a} ; a=1, \ldots, N\right\}$ are known on a node set $X=\left\{\mathbf{x}_{a}, a=1, \ldots, N\right\} \subset \mathbb{R}^{d}$. Without loss of generality, we assume that the affine hull of the node set is $\mathbb{R}^{d}$. We wish to construct approximations to $u$ of the form

$$
u^{h}(\mathbf{x})=\sum_{a=1}^{N} p_{a}(\mathbf{x}) u_{a}
$$

where the functions $p_{a}: \Omega \rightarrow \mathbb{R}$ will be referred to as shape functions. A particular choice of shape functions defines an approximation scheme. We shall require the shape functions to satisfy the zeroth- and first-order consistency conditions:

$$
\begin{aligned}
\sum_{a=1}^{N} p_{a}(\mathbf{x})=1 & \forall \mathbf{x} \in \Omega \\
\sum_{a=1}^{N} p_{a}(\mathbf{x}) \mathbf{x}_{a}=\mathbf{x} & \forall \mathbf{x} \in \Omega
\end{aligned}
$$

These conditions guarantee that affine functions are exactly reproduced by the approximation scheme. In general, the shape functions are not uniquely determined by the consistency conditions if $N>d+1$.

In addition, we shall require the shape functions to be non-negative, i.e.

$$
p_{a}(\mathbf{x}) \geqslant 0 \quad \forall \mathbf{x} \in \Omega, \quad a=1, \ldots, N
$$



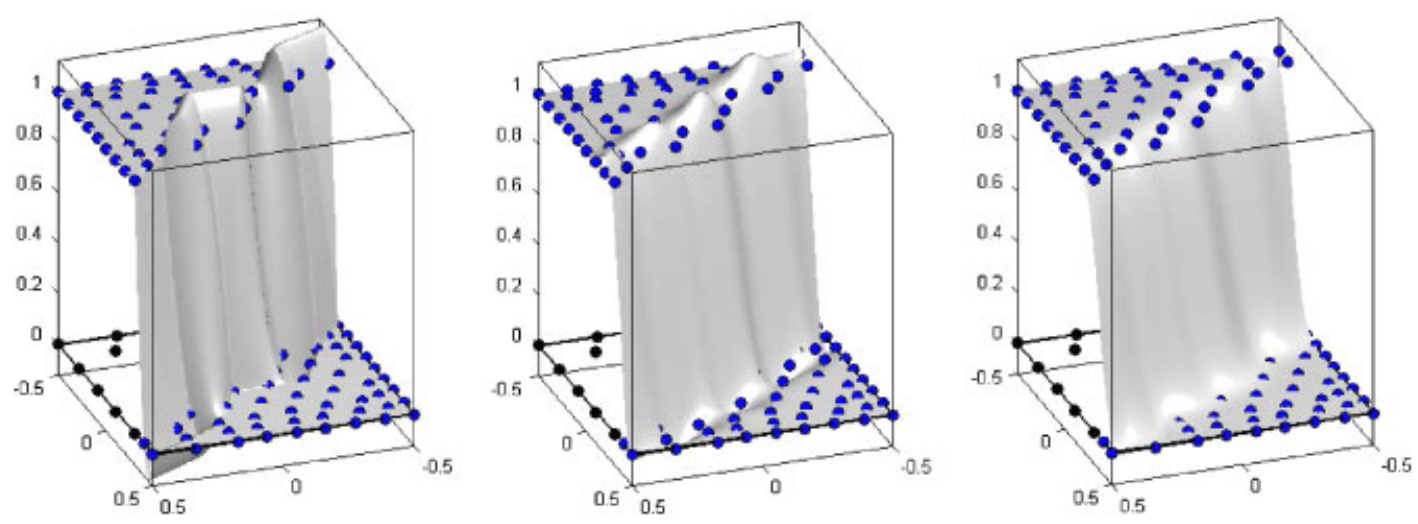

Figure 1. Non-monotonic behavior of the triangular quadratic finite element approximation (left) and the MLS second-order approximation (center) compared with the monotonic behavior of convex second-order approximants (described later, right) for discontinuous data.

The non-negativity of the shape functions, the partition of unity property and the first-order consistency conditions allow us to interpret the shape functions as generalized barycentric coordinates. This viewpoint is common in geometric modelling, e.g. in Bézier, B-Spline techniques [13], natural neighbor approximations [14], and subdivision approximations [15]. Non-negative linearly consistent approximants have long been studied in the literature [5]. These methods often present a number of attractive features, such as the related properties of monotonicity, the variation diminishing property (the approximation does not create extrema not present in the data), or smoothness preservation [16], of particular interest in the presence of shocks or sharp gradients. The nonoscillatory behavior for discontinuous data of the approximants presented here contrasts with the behavior of second-order triangular finite elements or second-order MLS approximants, as exemplified in Figure 1. Additionally, the non-negativity of the approximants allows us to interpret the shape functions at any given evaluation point as a discrete probability distribution. It follows from (2a), (2b) and (3) that the shape functions at $\mathbf{x} \in \operatorname{conv} X$ define a convex combination of vertices that evaluates to $\mathbf{x}$. In view of this property, we refer to non-negative and first-order consistent approximation schemes as convex approximation schemes [1].

The LME approach to building approximation schemes is to choose selected elements among all convex approximation schemes at a point $\mathbf{x}$, which we denote by

$$
\mathscr{P}_{\mathbf{x}}(X)=\left\{\mathbf{p} \in \mathbb{R}_{+}^{N} \mid \sum_{a=1}^{N} p_{a} \mathbf{x}_{a}=\mathbf{x}, \sum_{a=1}^{N} p_{a}=1\right\}
$$

where $\mathbf{p}(\mathbf{x})$ denotes the vector of $\mathbb{R}^{N}$ whose components are $\left\{p_{1}(\mathbf{x}), \ldots, p_{N}(\mathbf{x})\right\}$ and $\mathbb{R}_{+}^{N}$ is the non-negative orthant. Note that in the definition of the set of approximants $\mathscr{P}_{\mathbf{x}}(X)$ at a particular point $\mathbf{x}$, the explicit dependence of $\mathbf{p}$ on position is dropped. By direct comparison between the above-defined set of convex approximants and the convex hull of the node set

$$
\operatorname{conv} X=\left\{\mathbf{x} \in \mathbb{R}^{d} \mid \mathbf{x}=\sum_{a=1}^{N} \lambda_{a} \mathbf{x}_{a}, \lambda_{a} \geqslant 0, \sum_{a=1}^{N} \lambda_{a}=1\right\}
$$

it follows that the set of convex approximants $\mathscr{P}_{\mathbf{x}}(X)$ is non-empty if and only if $\mathbf{x} \in \operatorname{conv} X$. 
It was shown in [1] that any element of the set $\mathscr{P}_{\mathbf{x}}(X)$, in particular B-Spline or NURBS approximants, but not MLS approximants, satisfies a weak delta-Kronecker property from the outset. This property states that the shape functions of interior nodes vanish at the boundary of the domain, at extreme points (vertices) the delta-Kronecker property holds, and at any given face, only the shape functions of nodes in that face contribute to the approximation space. The reader is referred to the previous reference for details.

The LME approximants are the elements of $\mathscr{P}_{\mathbf{X}}(X)$ that minimize an information free-energy

$$
f_{\beta}(\mathbf{p} ; \mathbf{x})=\beta \sum_{a=1}^{N} p_{a}\left|\mathbf{x}-\mathbf{x}_{a}\right|^{2}+\sum_{a=1}^{N} p_{a} \log p_{a}
$$

These approximants are an optimal compromise between information-theory optimality and locality. The parameter $\beta \in \mathbb{R}_{+}$determines the degree of locality of the approximants, and one can rigorously show that in the limit $\beta \rightarrow+\infty$ the Delaunay linear approximants are recovered.

\section{SME APPROXIMANTS IN 1D}

In this section, we present the fundamental feasibility conditions required to build second-order convex approximants, and propose a method to deal with it in 1D. As we shall see, the treatment of higher-dimensional cases hinges on the ideas presented below.

\subsection{Feasibility conditions for second-order convex schemes}

An obvious generalization of the first-order convex schemes introduced in [1] is to further require higher-order consistency conditions, and still require the approximation scheme to be defined throughout the convex hull given by Equation (5). Adding naively a second-order consistency condition to Equation (4), the set of all second-order convex approximation schemes at the point $x$ can be written in $1 \mathrm{D}$ as

$$
\left\{\mathbf{s} \in \mathbb{R}_{+}^{N} \mid \sum_{a=1}^{N} s_{a} x_{a}^{2}=x^{2} \sum_{a=1}^{N} s_{a} x_{a}=x, \sum_{a=1}^{N} s_{a}=1\right\}
$$

It was proven in [1] that this set is empty unless $x=x_{a}$. This fact can be easily understood by rewriting the second-order condition as $\sum_{a} s_{a}\left(x-x_{a}\right)^{2}=0$, which in general cannot hold with $s_{a} \geqslant 0$ and $\sum_{a} s_{a}=1$. It should be emphasized that the consistency conditions can adopt a number of equivalent forms, as long as second-order polynomials can be exactly reproduced. If we insist in keeping the zeroth- and first-order conditions unchanged (partition of unity and barycentric coordinate property), we consider sets of convex approximants of the form

$$
\mathscr{S}_{x}(X)=\left\{\mathbf{s} \in \mathbb{R}_{+}^{N} \mid \sum_{a=1}^{N} s_{a}\left(x-x_{a}\right)^{2}=g(x), \sum_{a=1}^{N} s_{a} x_{a}=x, \sum_{a=1}^{N} s_{a}=1\right\}
$$

where $g(x)$ is a function to be specified making $\mathscr{S}_{x}(X)$ a non-empty set throughout conv $X$ and not destroying the second-order consistency, e.g. it might be a constant function or an affine one. As discussed in [1], it follows from the definition of the convex hull that $\mathscr{S}_{x}(X)$ is non-empty for any value of $x$ in conv $X$ if and only if

$$
\mathbf{y}=\left(x, x^{2}+g(x)\right)^{\mathrm{T}} \in \operatorname{conv} Y
$$




\section{J. CYRON, M. ARROYO AND M. ORTIZ}
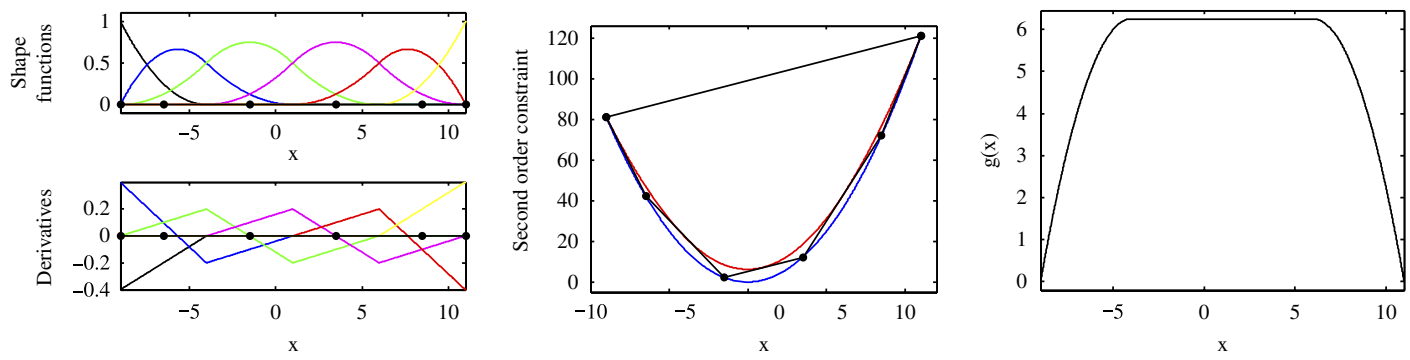

Figure 2. B-spline shape functions and their derivatives (left); moment space (black polygon), $x^{2}$ function (lower curve) and the second-order-constraint satisfied by the B-splines (upper curve enclosed by the polygon) (center); gap function $g(x)$ of the B-splines (right).

where the augmented node set $Y=\left\{\left(x_{1}, x_{1}^{2}\right),\left(x_{2}, x_{2}^{2}\right), \ldots,\left(x_{N}, x_{N}^{2}\right)\right\} \subset \mathbb{R}^{2}$ has been introduced. The crucial question is how to choose $g(x)$, which we will refer to as the gap function, in such a way that this feasibility condition is satisfied while second-order consistency is maintained.

To motivate the proposed method, let us look at an example of a convex, second-order approximation scheme, the quadratic B-Spline basis illustrated in Figure 2. In the left plot, the shape functions are shown. These non-negative functions are $C^{1}$ piecewise quadratic functions, and they can reproduce exactly up to second-order polynomials. The nodes $\left\{x_{1}, \ldots, x_{N}\right\}$ associated with these shape functions can be defined by the relation $\sum_{a} N_{a, 2}(x) x_{a}=x$, although these objects are not usually introduced in the B-Spline literature. The points of discontinuity of the second derivatives, the knots, are clearly noticeable in the plot of the derivatives of the shape functions. The center plot in Figure 2 shows the so-called moment space conv $Y$ (black polygon). Since B-splines are convex approximation schemes, it is obvious from the previous paragraph that the graph of the function $\sum_{a} N_{a, 2}(x) x_{a}^{2}=x^{2}+g(x)$ (upper curve, enclosed by the black polygon) lies within conv $Y, \forall x \in \operatorname{conv} X$. The figure also shows that the naive choice $g(x)=0$ is in general infeasible; in that the graph of $x^{2}$ (lower curve) does not lie within conv $Y$. Thus, B-splines are convex secondorder schemes for a specific choice $g(x)$ shown in the right plot of Figure 2. This gap function $g(x)$ is constant (with a value of $h^{2} / 4, h$ being the nodal spacing inside the domain) within the interior knots, while between a boundary knot and the following knot inside the domain, $g(x)$ is the unique second-order polynomial that vanishes on the boundary and keeps the $C^{1}$ continuity. Thus, the second-order constraint is a $C^{1}$ piecewise second-order polynomial function and it is easy to reproduce the function $x^{2}$ between two consecutive knots by linearly combining the three consistency conditions. Note carefully that the knots, where the shape functions have discontinuous second derivatives, are precisely the points where $\sum_{a} N_{a, 2}(x) x_{a}^{2}=x^{2}+g(x)$ touches the boundary of the moment space conv $Y$.

The example above illustrates how it is possible to construct non-empty sets of second-order convex approximants $\mathscr{S}_{x}(X)$ if we are willing to replace the canonical second-order consistency condition by a relaxed one with $g(x) \neq 0$. It also illustrates how the gap function of B-Spline is a particular choice (essentially constant except near the boundaries), and how there is room for alternative definitions of non-empty sets $\mathscr{S}_{x}(X)$. Once such set is fixed, in general there exist multiple approximants fulfilling the constraints, and max-ent can be used as a selection principle. A piecewise polynomial definition of the gap function is a natural and convenient choice on 1D, but is not easily generalizable to multiple dimensions, general node sets and general domains. 
We propose next a method to build the gap function from the first-order LME approximants, and to use the reduced face property to step from $n$ dimensions to $n+1$ dimensions as will be outlined in Section 4.1.

\subsection{Max-ent gap functions}

Figure 3 illustrates the effect of defining a constant gap function $g(x)$. While in the interior of the domain the curve $f(x)$ (continued curve), representing a vertical shift of $x^{2}$ (dashed curve), lies within the moment space (dashed trapezoid) and consequently $\mathscr{S}_{x}(X)$ is non-empty, the feasibility condition is violated near the ends of the domain, here $x_{1}$ and $x_{4}$. In order to describe a gap function fully compatible with the constraints, which in particular vanishes at the domain boundary, we consider functions of the form

$$
g(x)=\sum_{a=1}^{N} p_{\text {lin }, a}(x) w_{a}, \quad w_{a} \in \mathbb{R}
$$

where $p_{\operatorname{lin}, a}(x)$ are the first-order LME shape function related to node $a$ and $w_{a}$ is a weight to be determined. As discussed later, the smoothness of the second-order approximants obtained by invoking max-ent is at most that of $g(x)$. Since the LME approximants are $C^{\infty}$, the above choice does not hurt in any way the smoothness of the second-order approximants. Choosing a piecewise polynomial gap function with $C^{1}$ continuity as is done in quadratic B-Spline reduces the regularity of the approximants, and is not easily generalizable to higher dimensions.

We consider a node set $X=\left\{x_{1}, \ldots, x_{N}\right\}$ whose boundary nodes are $x_{1}$ and $x_{N}$. The choice $w_{1}=0, w_{N}=0$ and $w_{1<i<N}=g_{\text {int }}$ leads to a gap function with constant value $g_{\text {int }}$ nearly everywhere, except near the boundary where it smoothly ramps down to zero. This follows from the partition of unity, the locality, and the weak Kronecker-delta property of LME approximants. A typical example of such a gap function is depicted in Figure 3 (right). It is apparent that its graph lies within the convex hull spanned by the points $\left\{\left(x_{1}, w_{1}\right), \ldots,\left(x_{N}, w_{N}\right)\right\}$.

We provide next heuristics on the choice of $g_{\text {int }}$, which give in practice excellent results. The setup for a non-uniform node distribution is shown in Figure 4 (left), where the moment space is drawn with a dashed black line, and the $x^{2}$ graph in continued line. The gap function is best visualized by subtracting $x^{2}$ to the left-hand-side plot. For feasibility, the gap function should lie within the modified moment space in the center plot of Figure 4, delimited in its upper part by

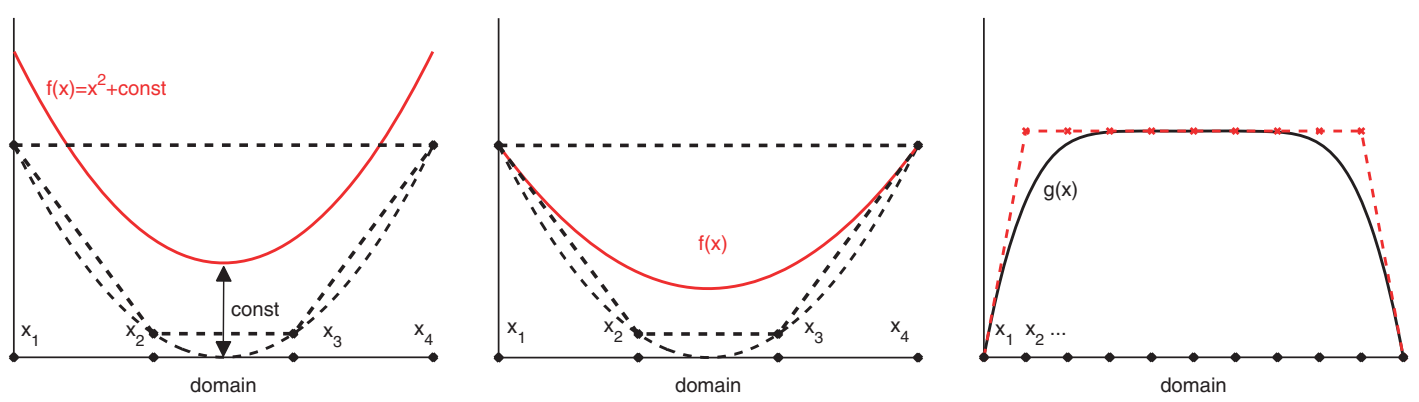

Figure 3. Domain with four nodes, related moment space (dashed trapezoid) and $f(x)$ (continued line) based on constant gap function (left) and max-ent gap function (middle); graph of a typical max-ent gap function (continued) and related convex hull (dashed) in a 1D domain with 11 nodes (right). 


\section{J. CYRON, M. ARROYO AND M. ORTIZ}
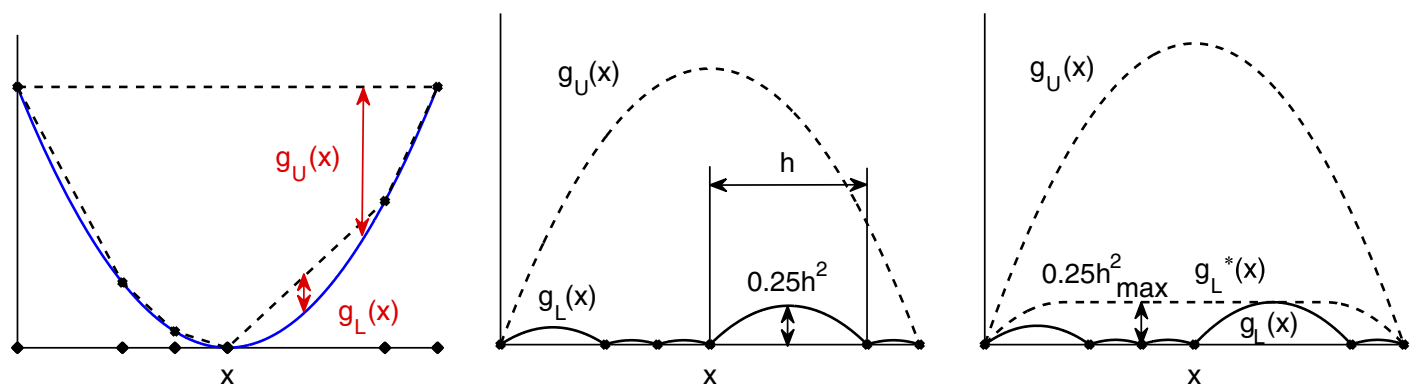

Figure 4. Upper boundary $g_{\mathrm{U}}(x)$ and lower boundary $g_{\mathrm{L}}(x)$ and $g_{\mathrm{L}}^{*}(x)$ for the gap function $g(x)$.

a concave parabola $g_{\mathrm{U}}(x)$, which vanishes at the ends of the interval, and by a function $g_{\mathrm{L}}(x)$ composed of quadratic bubbles spanning two consecutive nodes. The maximum of each of these bubbles is $h^{2} / 4$, where $h$ is the inter-node spacing. By insisting on a constant value of the gap function in the interior of the domain, we clearly require that $g_{\text {int }}=w_{1<i<N} \geqslant h_{\max }^{2} / 4$. For technical reasons, we introduce the function $g_{\mathrm{L}}^{*}(x)$, with constant value $h_{\max }^{2} / 4$ in the interior, and parabolic parts in the intervals $\left[x_{1} ; x_{1}+h / 2\right]$ and $\left[x_{N}-h / 2 ; x_{N}\right]$, so that the function remains $C^{1}$ and vanishes at the boundaries. It is obvious from this definition that $g_{\mathrm{L}}^{*} \geqslant g_{\mathrm{L}}$.

Let us look carefully at the feasibility $g_{\mathrm{L}}(x) \leqslant g(x) \leqslant g_{\mathrm{U}}(x)$ near the ends of the domain. Given that the gap function $g(x)$ is based on linear max-ent functions for which an explicit formula is not available, this requires further analysis. Let us consider a uniform point distribution for simplicity. Close to the boundary, in the intervals $\left[x_{1} ; x_{1}+h / 2\right]$ and $\left[x_{N}-h / 2 ; x_{N}\right]$, we have

$$
g_{\mathrm{L}}^{*}(\xi)=-\xi(\xi-h) \quad \text { with } 0 \leqslant \xi \leqslant \frac{h}{2}
$$

where $\xi$ is the distance from a point $x$ to the neighboring boundary node, $x_{1}$ or $x_{N}$. As shown in the forthcoming work, the linear max-ent shape functions related with boundary nodes $p_{\text {lin }, b d}(\xi)$ have the following bound for small $\xi$ :

$$
p_{\text {lin }, b d}(\xi) \leqslant \frac{h}{\xi+h}
$$

Consequently, letting

$$
w_{a}=\frac{\alpha h^{2}}{4}, \quad a=2, \ldots, N-1
$$

and recalling the partition of unity of the LME approximants, close to the boundary we have

$$
g(\xi) \geqslant \frac{\alpha h^{2}}{4}\left(1-\frac{h}{\xi+h}\right)=\frac{\alpha h^{2}}{4} \frac{\xi}{\xi+h}
$$

Comparing the Equations (10) and (12), it follows that the condition

$$
\xi^{2} \geqslant h^{2}\left(1-\frac{\alpha}{4}\right)
$$

ensures that for small $\xi, g(\xi) \geqslant g_{\mathrm{L}}^{*}(\xi)$ and hence $g(\xi) \geqslant g_{\mathrm{L}}(\xi)$. This condition holds true for all $0 \leqslant \xi \leqslant h / 2$ if and only if $\alpha \geqslant 4$. Consequently the interior gap weights $w_{1<i<N}=h^{2}$ ensure the 
condition $g(x) \geqslant g_{\mathrm{L}}(x)$ throughout the domain. Note carefully that the bound in Equation (11) is not tight, and in practice lower values of $\alpha$ guarantee feasibility throughout the domain.

Moving to the upper part of the moment space, the conditions for $g(x) \leqslant g_{\mathrm{U}}(x)$ follow from the convex hull property highlighted in Figure 3, and reduce to

$$
\frac{\alpha h^{2}}{4} \leqslant g_{U}\left(-\frac{L}{2}+h\right)
$$

In this equation we have assumed without loss of generality that $x_{1}=-L / 2$, where $L$ is the length of the domain. Since then

$$
g_{\mathrm{U}}(x)=-\left(x+\frac{L}{2}\right)\left(x-\frac{L}{2}\right)
$$

the upper feasibility condition is satisfied if

$$
L \geqslant h\left(1+\frac{\alpha}{4}\right)
$$

It is clear that for moderate values of $\alpha$, which are employed in practice, this condition poses no serious restriction.

We have assumed in the simple analysis above that the node distribution is uniform. If not, one can simply replace $h$ by $h_{\max }$ and retain feasibility. However, as shown below, the magnitude of the gap determines the spread of the shape functions; hence, such a choice leads to non-uniform locality of the approximants. Following the gap representation in Equation (9), spacial variations of the gap consisting of locally constant or affine functions with narrow non-polynomial transitions can be easily represented. The implementation of these ideas is postponed to future work. In the present paper, the max-ent gap function in Equation (9) is not a constant function near the ends of the domain; hence, the reproducing condition built into the approximants is not a second-order polynomial in these small regions. Consequently, an arbitrary second-order polynomial is not in general exactly reproduced by the approximants presented here in a narrow region near the boundary of the domain, but rather approximated by a smooth function. However, as the discretization is refined, the region where the second-order consistency condition is violated becomes smaller and smaller. The numerical experiments presented here in one and higher dimensions show that this fact does not affect the expected rate of convergence in the Galerkin solution of partial differential equations (PDEs). This observation is not obvious a priori; hence, there is room for a careful convergence analysis.

\subsection{SME approximants}

With the discussion in the previous section, a gap function $g(x)$ can be defined such that the set of second-order convex approximants

$$
\mathscr{S}_{x}(X)=\left\{\mathbf{s} \in \mathbb{R}_{+}^{N} \mid \sum_{a=1}^{N} s_{a}\left(x-x_{a}\right)^{2}=g(x), \sum_{a=1}^{N} s_{a}\left(x-x_{a}\right)=0, \quad \sum_{a=1}^{N} s_{a}=1\right\}
$$

is non-empty. Now the question is how to select approximants in these sets for each value of $x$. For this, we turn to max-ent as the selection principle [1]. Thus, we seek the SME approximants 


\section{J. CYRON, M. ARROYO AND M. ORTIZ}

$\mathbf{s}^{*}(x)$ as the unique solution of the convex program

$$
\begin{array}{ll}
\text { (SME) maximize } & H(\mathbf{s})=-\sum_{a=1}^{N} s_{a} \log s_{a} \\
\text { subject to } & s_{a} \geqslant 0, a=1, \ldots, N \\
& \sum_{a=1}^{N} s_{a}=1, \quad \sum_{a=1}^{N} s_{a}\left(x-x_{a}\right)=0 \\
& \sum_{a=1}^{N} s_{a}\left(x-x_{a}\right)^{2}=g(x)
\end{array}
$$

From an information theoretical viewpoint, this represents the least-biased choice consistent with the available information, here the reproducing conditions. From a practical viewpoint, max-ent is a convenient selection principle providing a semi-explicit exponential formula for the approximants. The Lagrange multipliers related to the first- and second-order consistency conditions can be obtained through duality from a strictly convex unconstrained optimization problem. Indeed, following the methods described in [1], we define the partition function

$$
Z(x, \lambda, \mu)=\sum_{a=1}^{N} \exp \left\{\lambda\left(x-x_{a}\right)+\mu\left[\left(x-x_{a}\right)^{2}-g(x)\right]\right\}
$$

The unique solution of the program (SME) is given by

$$
s_{a}^{*}(x)=\frac{1}{Z\left(x, \lambda^{*}(x), \mu^{*}(x)\right)} \exp \left\{\lambda^{*}(x)\left(x-x_{a}\right)+\mu^{*}(x)\left[\left(x-x_{a}\right)^{2}-g(x)\right]\right\}
$$

where $a=1, \ldots, N$ and

$$
\left\{\lambda^{*}(x), \mu^{*}(x)\right\}=\arg \min _{\lambda, \mu} \log Z(x, \lambda, \mu)
$$

The dual program above can be solved by Newton's method as discussed later.

The smoothness of the SME approximants can be precisely established through the implicit function theorem. Following the same argument as in [1], if the Slater condition is met, in the sense that there exists at least one strictly feasible element in $\mathscr{S}_{x}(X)$, then the approximants are as smooth as $g(x)$. With the max-ent gap function in Equation (9), this results in the SME approximants being $C^{\infty}$. From the previous section, the Slater condition holds if and only if the graph of the function $x^{2}+g(x)$ lies in the interior of the moment space defined as conv $\left\{\left(x_{1}, x_{1}^{2}\right),\left(x_{2}, x_{2}^{2}\right), \ldots,\left(x_{N}, x_{N}^{2}\right)\right\}$. This immediately shows that the max-ent selection principle combined with the feasible quadratic B-Spline gap does not lead to smooth approximants since the imposed second-order constraint touches the moment space precisely at the locations of the breakpoints. Figure 5 illustrates the similarity between B-splines and max-ent shape functions based on the same gap function.

Figure 6 illustrates the SME approximants obtained by using a max-ent gap function similar to Equation (9), but with a minor modification close to the boundary that allows a wider choice for $\alpha$. This modification uses a different node set, slightly refined near the boundaries, for the LME shape functions used in Equation (9). In the previous section, the analysis has been done with identical node sets for the LME and the SME approximants to keep the discussion as simple 

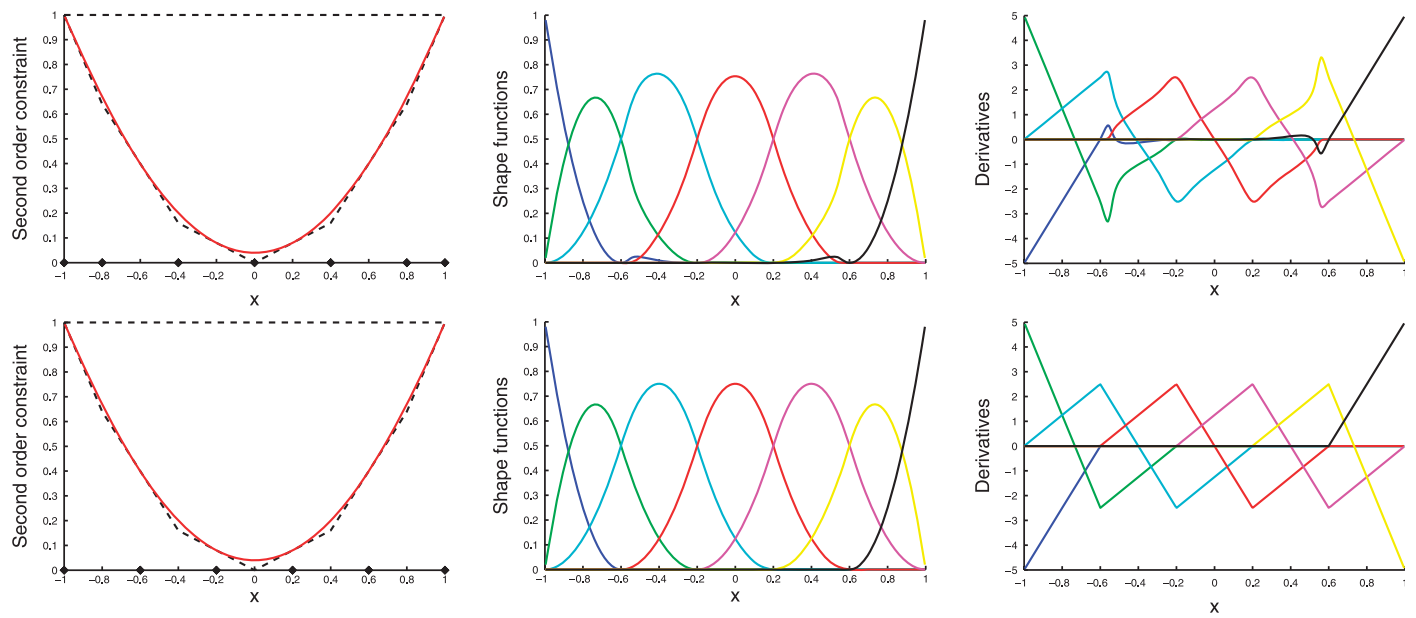

Figure 5. Second-order max-ent shape functions (top) based on the same gap function as B-spline functions (bottom). The black diamonds represent the SME nodes and B-Spline knots, respectively (note that the number of knots for B-Splines is by one smaller than the number of the max-ent nodes, which are the counterpart of the B-Spline control points). Apparently, particularly in the interior of the domain the max-ent shape functions replicate very closely the B-spline functions [correction made here after initial online publication].

as possible. It can be observed on the left that the graphs of $x^{2}+g(x)$ (continued lines) lie in the interior of the moment space, represented by dashed black lines. Consequently, the approximants are $C^{\infty}$. From Equation (22), it follows that the decay of the shape functions is Gaussian, which can be observed in the figure. Note that in the first-order LME approximants, it was necessary to introduce explicitly a bias in the entropy maximization to obtain local approximants, which also exhibited Gaussian decay. Here, the decay is an immediate consequence of the second-order consistency condition. As discussed in [1], even though the support of the shape functions is not strictly compact, the Gaussian decay is fast enough to consider from a numerical standpoint the approximants as compactly supported. The figure also exemplifies that the larger the gap, the larger is the support of the shape functions. This fact is more clearly depicted in Figure 7, where a non-uniform node distribution and a constant gap in the interior of the domain with value $h_{\max }^{2}$ have been considered. It is apparent that in regions where the gap, i.e. the difference between $x^{2}+g(x)$ and the lower part of the moment space, is larger, that is the left part of the domain, the shape functions have a larger support relative to the nodal spacing.

\section{SME APPROXIMANTS IN HIGHER DIMENSIONS}

We now turn to multiple dimensions. We restrict our attention to convex polyhedral domains. Nonconvex polyhedral domains are subsets of the domains we are considering, hence approximants for such domains are particular cases that nevertheless do not have the delta Kronecker property in the non-convex parts of the boundary. The treatment of curved complex boundaries will be dealt with in future work. 


\section{J. CYRON, M. ARROYO AND M. ORTIZ}
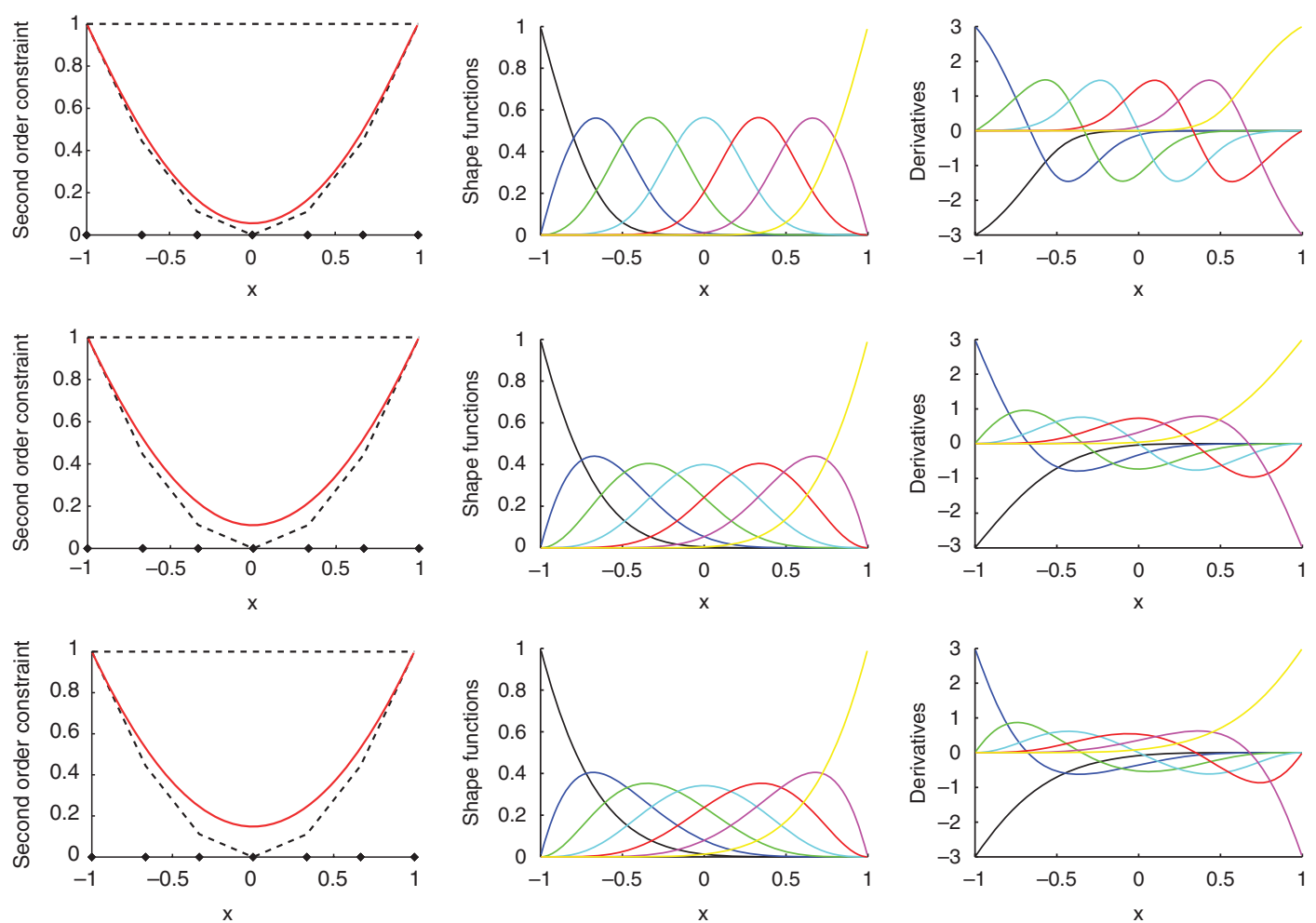

Figure 6. Second-order max-ent shape functions (middle), their derivatives (right) and the second-order constraint (left) they satisfy are plotted for different amplitudes of the gap function $g_{\text {int }}=0.5 h^{2}$ (top), $g_{\text {int }}=h^{2}$ (middle), $g_{\text {int }}=1.5 h^{2}$ (bottom). Obviously the shape functions spread out the further in the domain, the larger the gap amplitude.

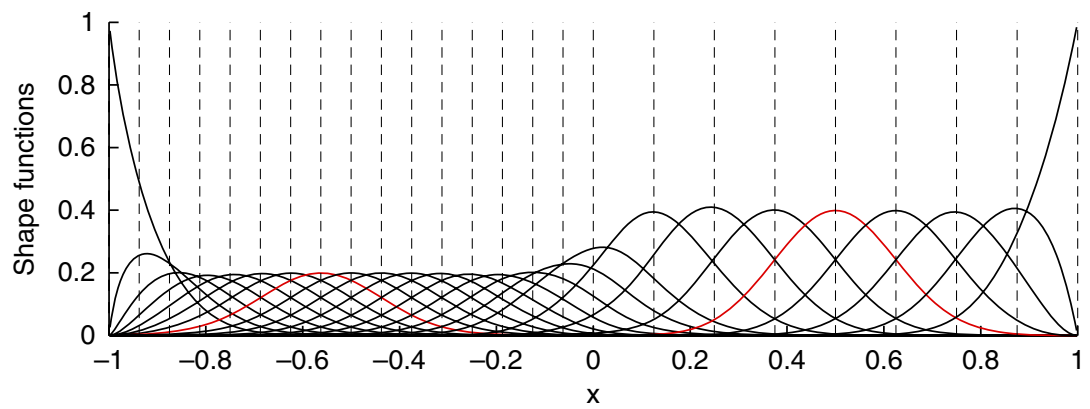

Figure 7. Second-order max-ent shape functions with non-uniform discretization (the node positions are indicated with vertical lines). 


\subsection{Second-order convex schemes in higher dimensions}

Generalizing from previous sections to higher dimensions, we consider the set of second-order convex schemes in $\mathbb{R}^{d}$

$$
\mathscr{S}_{\mathbf{x}}(X)=\left\{\mathbf{s} \in \mathbb{R}_{+}^{N} \mid \sum_{a=1}^{N} s_{a}\left(\mathbf{x}-\mathbf{x}_{a}\right)^{\otimes 2}=\mathbf{G}(\mathbf{x}), \sum_{a=1}^{N} s_{a} \mathbf{x}_{a}=\mathbf{x}, \quad \sum_{a=1}^{N} s_{a}=1\right\}
$$

where we use the notation $\mathbf{a} \otimes \mathbf{a}=\mathbf{a}^{\otimes 2}$ for the dyadic product of a vector with itself. The gap is now a matrix $\mathbf{G}(\mathbf{x})$, which from the expression above is necessarily symmetric and positive semidefinite. Furthermore, we will require this gap matrix to be positive definite in the interior of the domain to obtain $C^{\infty}$ approximants. Indeed, recalling the argument made earlier, the smoothness of the approximants requires Slater's conditions to hold, which implies that there exist strictly feasible elements of $\mathscr{S}_{\mathbf{x}}(X)$, i.e. strictly positive reals $s_{a}^{*}, a=1, \ldots, N$ satisfying the constraints. Consider now an arbitrary vector $\mathbf{u} \in \mathbb{R}^{d}$. Since the affine hull of the node set is $\mathbb{R}^{d}$, not all the values $u_{a}=\mathbf{u} \cdot\left(\mathbf{x}-\mathbf{x}_{a}\right)$ are zero. Consequently $\mathbf{u} \cdot \mathbf{G}(\mathbf{x}) \mathbf{u}=\sum_{a} s_{a}^{*} u_{a}^{2}>0$; hence, $\mathbf{G}(\mathbf{x})$ should be positive definite. At the boundary, Slater's condition cannot hold and the gap matrix is semi-definite positive.

As before, the gap matrix must be chosen in such a way that the feasibility conditions hold, and thus the set $\mathscr{S}_{\mathbf{X}}(X)$ is non-empty. Here, the feasibility conditions take the following mathematical expression:

$$
(\mathbf{x}, \mathbf{x} \otimes \mathbf{x}+\mathbf{G}(\mathbf{x})) \in \operatorname{conv}\left\{\left(\mathbf{x}_{a}, \mathbf{x}_{a} \otimes \mathbf{x}_{a}\right)\right\}_{1 \leqslant a \leqslant N} \quad \forall \mathbf{x} \in \operatorname{conv} X
$$

This condition involves points in the product space of vectors in $\mathbb{R}^{d}$ and symmetric matrices in $\mathbb{R}^{d \times d}$, which in $3 \mathrm{D}$ can be reduced to a convex hull query in $\mathbb{R}^{9}$ by means of the Voigt notation for the symmetric matrices.

Like in the $1 \mathrm{D}$ case, we choose to define a max-ent gap taking the form

$$
\mathbf{G}(\mathbf{x})=\sum_{a=1}^{N} p_{\operatorname{lin}, a}(\mathbf{x}) \mathbf{W}_{a}
$$

We build the procedure to set the weights $\mathbf{W}_{a}$ heuristically as before, and by increasing the dimension. To this end, given a unit vector $\mathbf{u} \in \mathbb{R}^{d}$, we define the projected node set as $X^{\mathbf{u}}=$ $\left\{x_{1}^{\mathbf{u}}, x_{2}^{\mathbf{u}}, \ldots, x_{N}^{\mathbf{u}}\right\}$, where $x_{a}^{\mathbf{u}}=\mathbf{u} \cdot \mathbf{x}_{a}$ and the projected evaluation point $x^{\mathbf{u}}=\mathbf{u} \cdot \mathbf{x}$. A necessary condition for the non-emptiness of $\mathscr{S}_{\mathbf{x}}(X)$ is the non-emptiness of the projected set of convex approximants, an instance of the sets investigated in Section 3.1:

$$
\mathscr{S}_{x^{\mathbf{u}}}\left(X^{\mathbf{u}}\right)=\left\{\mathbf{s} \in \mathbb{R}_{+}^{N} \mid \sum_{a=1}^{N} s_{a}\left(x^{\mathbf{u}}-x_{a}^{\mathbf{u}}\right)^{2}=g^{\mathbf{u}}\left(x^{\mathbf{u}}\right), \sum_{a=1}^{N} s_{a} x_{a}^{\mathbf{u}}=x^{\mathbf{u}}, \quad \sum_{a=1}^{N} s_{a}=1\right\}
$$

Here, $g^{\mathbf{u}}\left(x^{\mathbf{u}}\right)=\mathbf{u} \cdot \mathbf{G}(\mathbf{x}) \mathbf{u}=\sum_{a=1}^{N} p_{\operatorname{lin}, a}(\mathbf{x}) w_{a}^{\mathbf{u}}$, where $w_{a}^{\mathbf{u}}=\mathbf{u} \cdot \mathbf{W}_{a} \mathbf{u}$.

Consider a polygonal domain in 2D. A vertex or extreme point of this domain $\mathbf{x}_{b}$ is the end-point of two different faces, characterized by two non-collinear unit vectors $\mathbf{t}$ and $\mathbf{u}$. The feasibility condition at $\mathbf{x}_{b}$ implies that the sets $\mathscr{S}_{x_{b}^{\mathrm{t}}}\left(X^{\mathbf{t}}\right)$ and $\mathscr{S}_{x_{b}^{\mathbf{u}}}\left(X^{\mathbf{u}}\right)$ are non-empty. Recalling the results 


\section{J. CYRON, M. ARROYO AND M. ORTIZ}

in Section 3.1, and the fact that at a vertex $p_{\operatorname{lin}, a}\left(\mathbf{x}_{b}\right)=\delta_{a b}$, this requires that

$$
0=\mathbf{u} \cdot \mathbf{W}_{b} \mathbf{u}=\mathbf{t} \cdot \mathbf{W}_{b} \mathbf{t}
$$

Since the weight matrix is positive semi-definite and symmetric, this implies that at vertices $\mathbf{W}_{b}=\mathbf{0}$.

Consider now a 1 -face $F$ aligned with a unit vector $\mathbf{t}$. We know that within this face the only non-zero convex approximants are those whose nodes belong to the face (reduced face property [1]). Thus, for $x \in F$ the discussion on the set $\mathscr{S}_{x_{b}^{\mathrm{t}}}\left(X^{\mathbf{t}}\right)$ precisely reduces to the $1 \mathrm{D}$ case discussed in Section 3.1 for $F$ and its nodes. We conclude that

$$
\mathbf{t} \cdot \mathbf{W}_{b} \mathbf{t}=\frac{\alpha h^{2}}{4} \quad \text { for } \mathbf{x}_{b} \in F
$$

for an suitably chosen value of $\alpha$ discussed before, $h$ being the typical nodal spacing. Now, considering a normal vector $\mathbf{n}$ to $F$, the projection of the nodes in $F$ collapses to a single point, which is at the boundary of $\operatorname{conv} X^{\mathbf{n}}$, and consequently

$$
\mathbf{n} \cdot \mathbf{W}_{b} \mathbf{n}=0 \quad \text { for } \mathbf{x}_{b} \in F
$$

We can thus represent the weight matrix for points belonging to a 1-face as

$$
\mathbf{W}_{b}=\frac{\alpha h^{2}}{4} \mathbf{t} \otimes \mathbf{t}
$$

At interior points, assuming the node distribution does not present a strong anisotropy, it is reasonable to prescribe simply

$$
\mathbf{W}_{b}=\frac{\alpha h^{2}}{4} \mathbf{I d}
$$

As a matter of fact, it is easily understood that the real eigenvalues of $\mathbf{W}_{a}$ set the spread of the shape functions in the vicinity of $\mathbf{x}_{a}$ in the directions of the corresponding eigenvectors.

The 3D case proceeds analogously. For vertice, 1-faces and interior nodes, the arguments are identical. Again resorting to the reduced-face property of convex approximants, 2-faces reduce to the $2 \mathrm{D}$ case within their plane, while the weights should vanish in the normal direction. Selecting an orthonormal basis $\left\{\mathbf{t}_{1}, \mathbf{t}_{2}\right\}$ on the 2 -face under consideration, for nodes on this face we write

$$
\mathbf{W}_{b}=\frac{\alpha h^{2}}{4}\left(\mathbf{t}_{1} \otimes \mathbf{t}_{1}+\mathbf{t}_{2} \otimes \mathbf{t}_{2}\right)
$$

Although the above prescriptions do not substitute further mathematical study on the feasibility conditions of second- and higher-order convex approximants in multiple dimensions, we have extensively tested for many node sets in $2 \mathrm{D}$ and $3 \mathrm{D}$ the conditions in Equation (20) numerically by using the Qhull library. In all the cases, the set $\mathscr{S}_{\mathbf{x}}(X)$ has been found to be non-empty; hence, it makes sense to apply the max-ent selection principle.

\subsection{SME approximants}

For completeness, we present next the max-ent problem subject to the constraints encoded in $\mathscr{S}_{\mathbf{x}}(X)$, as well as the semi-explicit solution to this convex program using duality. For a derivation of the procedure see [1] or a standard text in convex optimization, e.g. [17]. At each point $\mathbf{x}$ in the domain, the second-order convex approximants are defined as the unique solution $\mathbf{s}^{*}(\mathbf{x})$ to the 
convex program

$$
\begin{aligned}
& \text { maximize } \quad H(\mathbf{s})=-\sum_{a=1}^{N} s_{a} \log s_{a} \\
& \text { subject to } s_{a} \geqslant 0, \quad a=1, \ldots, N \\
& \\
& \sum_{a=1}^{N} s_{a}=1, \quad \sum_{a=1}^{N} s_{a}\left(\mathbf{x}-\mathbf{x}_{a}\right)=\mathbf{0} \\
& \\
& \sum_{a=1}^{N} s_{a}\left(\mathbf{x}-\mathbf{x}_{a}\right) \otimes\left(\mathbf{x}-\mathbf{x}_{a}\right)=\mathbf{G}(\mathbf{x})
\end{aligned}
$$

Again, we define the partition function

$$
Z(\mathbf{x}, \boldsymbol{\lambda}, \boldsymbol{\mu})=\sum_{a=1}^{N} \exp \left\{\boldsymbol{\lambda} \cdot\left(\mathbf{x}-\mathbf{x}_{a}\right)+\boldsymbol{\mu}:\left[\left(\mathbf{x}-\mathbf{x}_{a}\right) \otimes\left(\mathbf{x}-\mathbf{x}_{a}\right)-\mathbf{G}(\mathbf{x})\right]\right\}
$$

The unique solution to the program (SME) is given by

$$
s_{a}^{*}(\mathbf{x})=\frac{1}{Z\left(\mathbf{x}, \lambda^{*}(\mathbf{x}), \boldsymbol{\mu}^{*}(\mathbf{x})\right)} \exp \left\{\lambda^{*}(\mathbf{x}) \cdot\left(\mathbf{x}-\mathbf{x}_{a}\right)+\boldsymbol{\mu}^{*}(\mathbf{x}):\left[\left(\mathbf{x}-\mathbf{x}_{a}\right) \otimes\left(\mathbf{x}-\mathbf{x}_{a}\right)-\mathbf{G}(\mathbf{x})\right]\right\}
$$

where $a=1, \ldots, N$ and

$$
\left\{\lambda^{*}(\mathbf{x}), \boldsymbol{\mu}^{*}(\mathbf{x})\right\}=\arg \min _{\lambda, \mu} \log Z(\mathbf{x}, \boldsymbol{\lambda}, \boldsymbol{\mu})
$$

Thus, at each point in space, the evaluation of the shape functions requires the solution of this strictly convex unconstrained optimization problem. The number of unknowns in $d$ dimensions is $d+d(d+1) / 2$. It can be efficiently solved by means of Newton's method, possibly combined with line search. With good initial guesses for $\lambda^{*}(\mathbf{x})$ and $\boldsymbol{\mu}^{*}(\mathbf{x})$, the line search can be avoided for efficiency and convergence is reached in a few iterations. For $\boldsymbol{\mu}$, the initial guess is related to the decay of the shape functions. For $\lambda$ in the interior of the domain, zero is a good initial guess.

The Newton iterations require the first and second derivatives of $\log Z(\mathbf{x}, \lambda, \boldsymbol{\mu})$ with respect to $\lambda$ and $\boldsymbol{\mu}$. Let us denote by $\partial_{\lambda} \log Z$ the partial derivatives of the function $Z(\mathbf{x}, \boldsymbol{\lambda}, \boldsymbol{\mu})$ with respect to the vector $\lambda$ arranged in the form of a column vector. Second derivatives are written analogously, e.g. $\partial_{\lambda \mu}^{2} \log Z$ is a third-order tensor whose imn-element denotes the partial derivative of $\log Z$ with respect to the $i$ th element of $\lambda$ and the $m n$-element of $\boldsymbol{\mu}$. In the computer implementation, the symmetry of $\boldsymbol{\mu}, \mathbf{G}$ and so on is exploited through the Voigt notation. In the equations below, we write $s_{a}(\mathbf{x})$ for the expression in Equation (22) evaluated at arbitrary values of the Lagrange multipliers. We then have

$$
\begin{aligned}
\partial_{\lambda} \log Z & =\sum_{a} s_{a}\left(\mathbf{x}-\mathbf{x}_{a}\right) \\
\partial_{\boldsymbol{\mu}} \log Z & =\sum_{a} s_{a}\left[\left(\mathbf{x}-\mathbf{x}_{a}\right)^{\otimes 2}-\mathbf{G}(\mathbf{x})\right] \\
\partial_{\lambda \lambda}^{2} \log Z & =\sum_{a} s_{a}\left(\mathbf{x}-\mathbf{x}_{a}\right)^{\otimes 2}-\left(\partial_{\lambda} \log Z\right)^{\otimes 2}
\end{aligned}
$$




$$
\begin{aligned}
& \partial_{\boldsymbol{\mu} \mu}^{2} \log Z=\sum_{a} s_{a}\left[\left(\mathbf{x}-\mathbf{x}_{a}\right)^{\otimes 2}-\mathbf{G}(\mathbf{x})\right]^{\otimes 2}-\left(\partial_{\boldsymbol{\mu}} \log Z\right)^{\otimes 2} \\
& \partial_{\lambda \boldsymbol{\mu}}^{2} \log Z=\sum_{a} s_{a}\left(\mathbf{x}-\mathbf{x}_{a}\right) \otimes\left[\left(\mathbf{x}-\mathbf{x}_{a}\right)^{\otimes 2}-\mathbf{G}(\mathbf{x})\right]-\left(\partial_{\lambda} \log Z\right) \otimes\left(\partial_{\boldsymbol{\mu}} \log Z\right)
\end{aligned}
$$

The values $\lambda^{*}(\mathbf{x})$ and $\boldsymbol{\mu}^{*}(\mathbf{x})$ have to satisfy the following condition:

$$
\begin{aligned}
& \partial_{\boldsymbol{\mu}} \log Z\left(\mathbf{x}, \lambda^{*}(\mathbf{x}), \boldsymbol{\mu}^{*}(\mathbf{x})\right)=0 \\
& \partial_{\lambda} \log Z\left(\mathbf{x}, \lambda^{*}(\mathbf{x}), \boldsymbol{\mu}^{*}(\mathbf{x})\right)=0
\end{aligned}
$$

Figure 8 shows two examples of the shape functions for an interior node in 2D. The smoothness of the approximants is apparent. It can also be observed how the gap matrix allows us to modulate the spread of the shape functions, and it is even easy to build anisotropic SME approximants by tuning the eigenvalues and eigenvectors of the gap matrix, similar to what was done for the first-order max-ent approximants in [1].

We note at this point that there is only a handful of available non-negative (convex) secondor higher-order approximation methods in multiple dimensions. Tensor product approximants can be used to generalize 1D methods such as B-Spline to multiple dimensions, but require structured grids. Recent examples include the References $[6,18]$. Subdivision approximations [15] can deal with unstructured data, but so far these approximants are theoretically well founded only in 2D. The proposed approach provides a general framework to construct higher-order non-negative approximants from unstructured scattered data in any spacial dimension.

\subsection{Imposing boundary values}

In some applications it is important to impose values to the approximation at the boundary of the domain. Approximation schemes that are interpolatory, i.e. that verify the Kronecker delta property, have no difficulties in this respect since they are sufficient to impose the boundary values at the nodal values. The same applies to approximants satisfying a weak Kronecker delta property
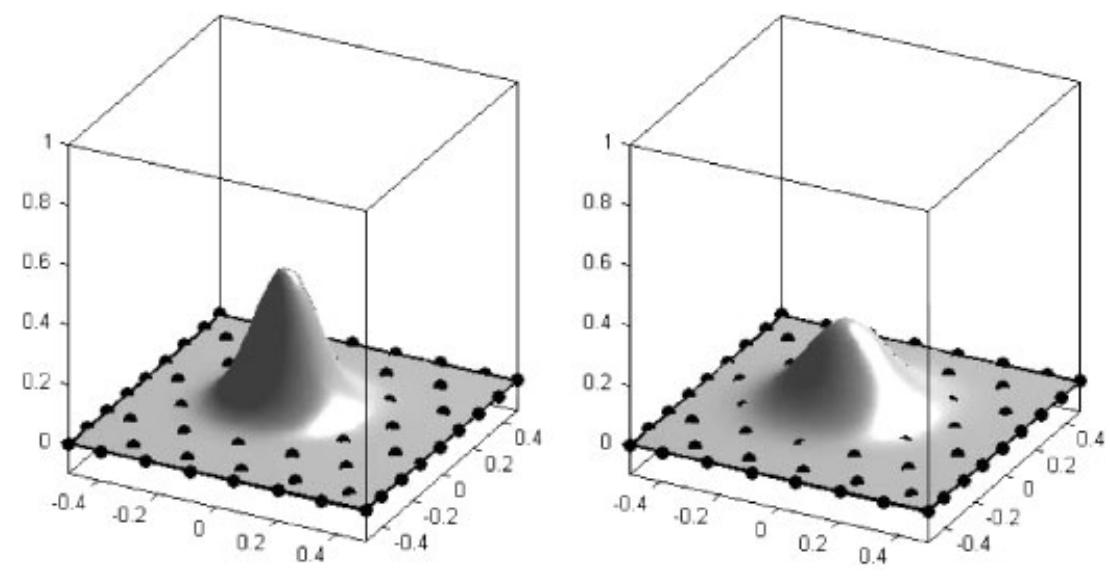

Figure 8. Illustration of the second order max-ent approximants in 2D; shape functions corresponding to two different gaps. 

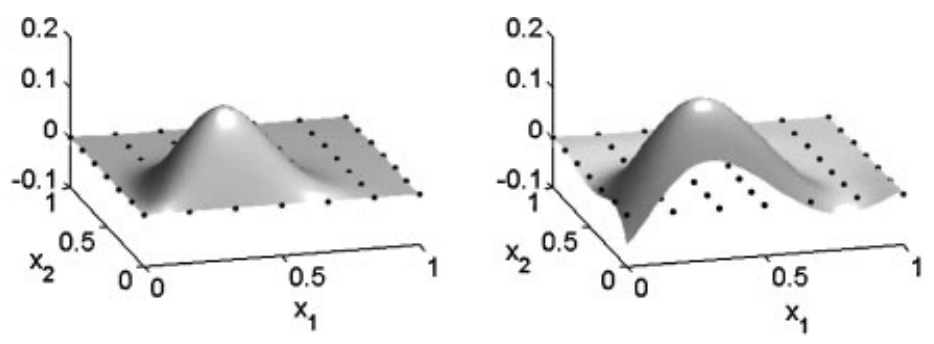

Figure 9. Second-order max-ent shape function (left) and MLS shape function (right) in two-dimensional domain.

such as the first-order LME approximants [1], where shape functions of interior nodes vanish at the boundary, and the only shape functions that are non-zero at a face are those of nodes on the face. This is also the case for B-Spline and NURBS approximants, which are instances of convex approximants. In contrast, typical meshfree methods based on the MLS approximants do not have these properties, and special methods need to be implemented to impose the Dirichlet boundary conditions. As reviewed in $[4,19]$, a number of methods have been proposed to impose essential boundary conditions in the numerical approximation of boundary value problems with noninterpolatory approximants. Penalty formulations present obvious drawbacks, while the Lagrange multipliers method requires a careful choice of the functional spaces to avoid over constraining the approximation or having stability problems. The Nitsche method has been proposed as a competitive alternative, but some conditions on the node arrangement and the penalty parameter must be met for convergence.

In Figure 9 an interior second-order MLS and SME shape function are plotted. One of the main features of convex approximants that follows from their non-negativity is that the approximation at the boundary does not depend on interior nodes, which clearly does not hold for MLS approximants. This illustrates how max-ent approximants unite advantages of meshfree methods such as high smoothness and flexibility, with advantages of finite element methods such as the ease to prescribe boundary data to the approximation.

\section{NUMERICAL EXAMPLES}

We present next the application of the meshfree max-ent approximants for the numerical approximation of PDEs by the Galerkin methods. We first present structural vibration examples taken from [7], and then we consider the solution of the Poisson equation in 2D and 3D. In all cases, we compare the results with those of the second-order Lagrange finite elements, B-Spline and MLS approximants.

\subsection{Vibrational analysis}

5.1.1. Eigenfrequencies of an elastic rod. We consider the vibrations of a linearly elastic rod with fixed ends, governed by

$$
\begin{aligned}
u^{\prime \prime}+\omega^{2} u & =0 \quad \text { in }(0,1) \\
u(0) & =u(1)=0
\end{aligned}
$$




\section{J. CYRON, M. ARROYO AND M. ORTIZ}

whose eigenfrequencies can be computed analytically as $\omega_{n}=n \pi, n=1,2,3, \ldots$. Numerically, we perform a Galerkin projection of these equations, and with $N$ degrees of freedom (DOF) and computing the numerical eigenfrequencies $\omega_{n}^{h}$, we define the ratios

$$
r\left(\omega_{n}\right)=\frac{\omega_{n}^{h}}{\omega_{n}}
$$

with $1 \leqslant n \leqslant N$. These ratios measure the numerical error across the spectrum. Similarly, we define a relative $L_{2}$ error norm by

$$
\|e\|_{\mathrm{rel}, L_{2}}=\left[\frac{1}{N} \sum_{n=1}^{N}\left(\frac{\omega_{n}^{h}}{\omega_{n}}-1\right)^{2}\right]^{1 / 2}
$$

Figure 10 (left) shows the ratio between the numerical and the exact frequencies for different approximation schemes, namely the second-order Lagrange finite elements (Lg2), quadratic BSplines (Bsp), second-order moving least squares (MLS), first-order max-ent (LME) and secondorder max-ent (SME). For low frequencies, all schemes provide accurate approximations to the spectrum, i.e. ratios close to one. The departure from the ideal behavior shows that discretization introduces dispersion. As pointed out in [7], the second-order Lagrange finite elements show an optical branch in the spectrum for higher frequencies corresponding to standing waves. This branch is spurious in the sense that the original continuous system has only an acoustical branch. The optical modes are well known in the lattice dynamics literature [20], and result here from the spacial inhomogeneity of the Lagrange quadratic shape functions, with cusped basis functions at the element ends and bubbles for interior nodes. The resulting high-frequency modes, if excited in a dynamical simulation, destroy the solution and special methods are needed to take care of them. As discussed in [7], B-Spline and related method produce much more accurate numerical spectra devoid of optical branches.
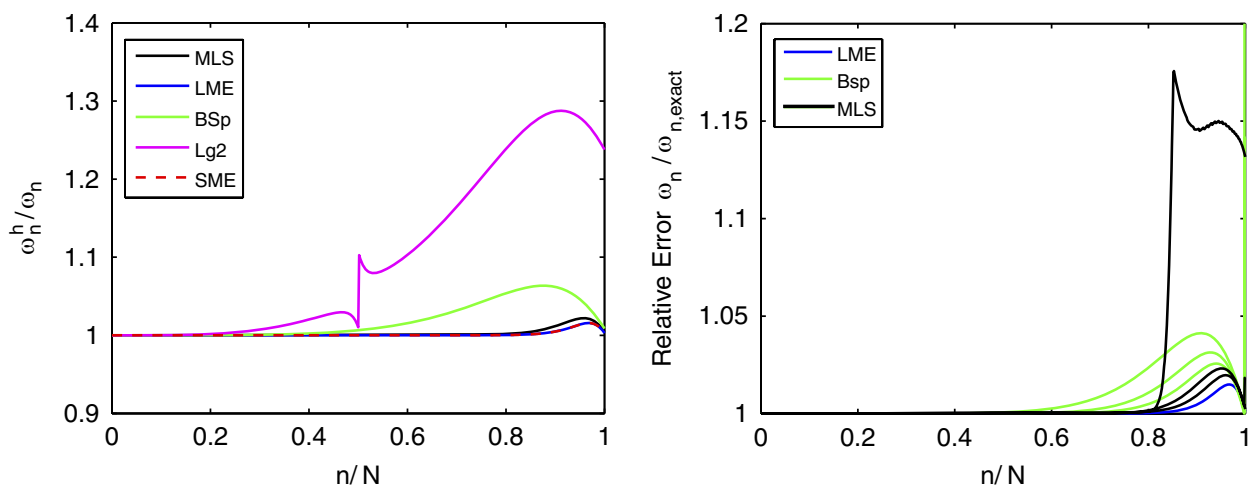

Figure 10. Relative error in the approximation of the eigenfrequency spectrum of an elastic rod for 999 degrees of freedom and different methods where an optimized dilation parameter for MLS is used (left); comparison between LME, higher order B-Splines (cubic, quartic, quintic) and MLS with different dilation parameters $(3.0,4.0$ and 4.5$)$; note carefully that for a dilation parameter of 3.0 or 4.0 , the MLS results are comparable with the LME whereas for a dilation parameter of 4.5 the MLS method produces very large high-frequency errors (right). 

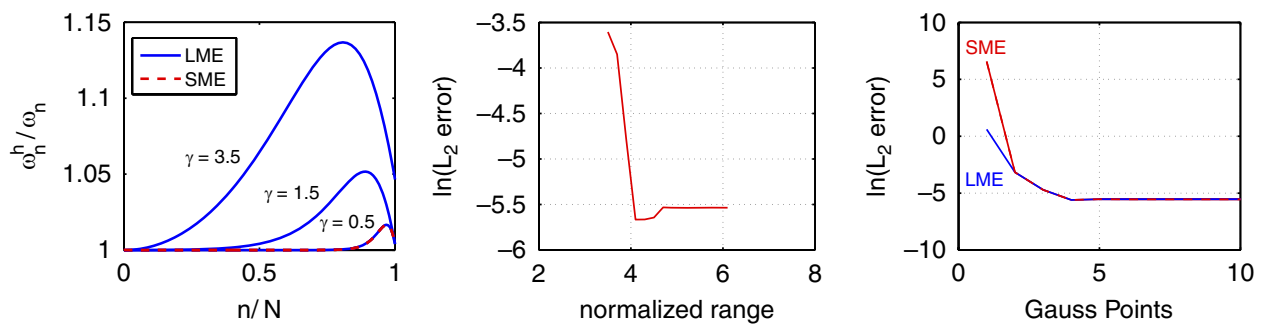

Figure 11. Comparison for the vibrations of a rod between second-order max-ent approximants and linear max-ent approximants with different locality factors (left), error dependence on the normalized search range of second-order max-ent functions (middle) and number of Gauss points necessary for proper numerical integration (right).

Figure 10 (left) shows the exceptional performance of meshfree methods in this respect, with much lower errors. For these methods, the frequency ratios cannot be distinguished from the one in this plot for more than $80 \%$ of the spectrum, while for B-Spline the discrepancy is noticeable in more than half of the spectrum. Figure 10 (right) shows that even the linear max-ent approximants are considerably more accurate for this problem than higher-order B-Spline (cubic, quartic and quintic). This seems to indicate that for vibration problems and the presented measure of accuracy, the smoothness or support size of the approximants plays a bigger role than the order of polynomial reproducibility. The figure also highlights a serious drawback of the MLS method, further discussed in the following sections. It is found that the results with MLS approximants are not robust with respect to the dilation parameter, which determines the support of the shape functions. Small variations of this parameter can turn a good behavior into inaccurate high-frequency results, with the emergence of spurious optical branches.

The left plot in Figure 11 shows the influence of the locality parameter in the spectrum of LME approximants. It can be observed that the more spread out they are (in some sense the smoother), the more accurate they are. For very low locality parameters, they closely replicate the spectrum of the SME approximants. Comparing the exponential forms of the LME and the SME approximants, it is conceivable that for a uniform node distribution, the locality parameter of LME is such that it closely mimics the Lagrange multiplier of the second-order constraint of SME, hence leading to very similar approximants. The good results of the MLS approximants is also noteworthy in Figure 10. However, as illustrated in the other examples, the quality of the MLS results highly depends on the choice of the dilation parameter. It is observed that for certain values a spurious optical branch appears, whereas SME approximation consistently produce accurate results.

From a practical viewpoint, it is interesting to point out two aspects of the implementation. Strictly speaking, the support of SME shape functions is the entire domain. However, as discussed in detail in [1], the fast Gaussian decay makes these approximants compactly supported to machine precision. It is computationally convenient to limit the neighbor search to a certain range. We define a normalized range $r_{\text {norm }}$ as the ratio between the search range $r$ and the characteristic discretization length $h, r_{\text {norm }}=r / h$. As shown in the center plot of Figure 11 a normalized range of five is sufficient in this example, although this depends on the choice of the magnitude of the gap function, i.e. the parameter $\alpha$ described before. Numerical quadrature is performed with the Gauss-Legendre rules, and the right plot of Figure 11 shows that four Gauss points per integration element (delimited by two nodes) were sufficient for integration of the SME functions. As expected, 


\section{J. CYRON, M. ARROYO AND M. ORTIZ}

this results in extra computational cost as compared with the B-Spline, which can be nevertheless outweighed by the extra accuracy granted by the SME approximants.

5.1.2. Eigenfrequencies of an Euler-Bernoulli beam. We perform now a similar analysis for the eigenfrequencies of an Euler-Bernoulli elastic beam

$$
\begin{aligned}
u^{\prime \prime \prime \prime}-\omega^{2} u & =0 \quad \text { in }(0,1) \\
u(0) & =u^{\prime \prime}(0)=u(1)=u^{\prime \prime}(1)=0
\end{aligned}
$$

As in the previous example, the ratio of numerical to exact eigenfrequencies, given by $\omega_{n}=(n \pi)^{2}$, is shown in Figure 12 for second-order B-Spline, MLS and max-ent approximants. Even though we are dealing with a fourth-order PDE, we also plot the results obtained with the first-order max-ent approximants. Again one can see that the meshfree methods outperform B-Splines, and among meshfree methods, SME approximants provide the most accurate results.

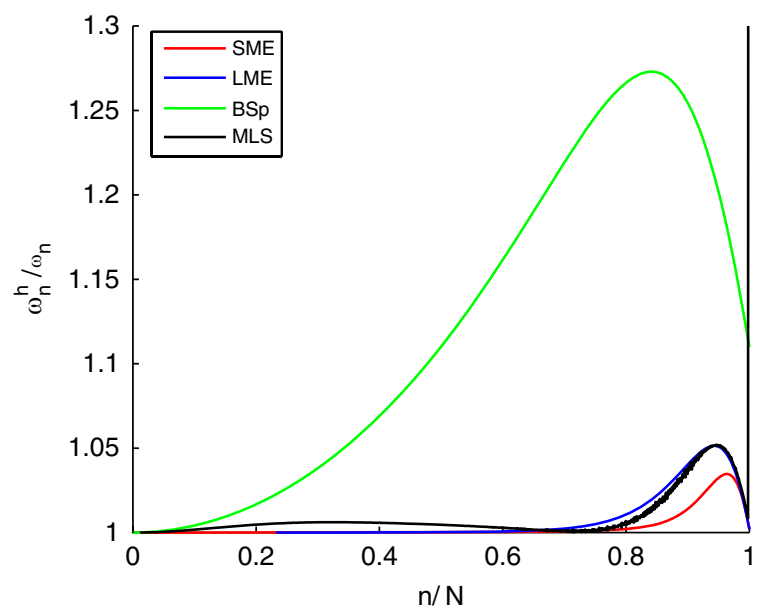

Figure 12. Relative error in the approximation of the eigenfrequency spectrum of an Euler-Bernoulli beam for 999 degrees of freedom.
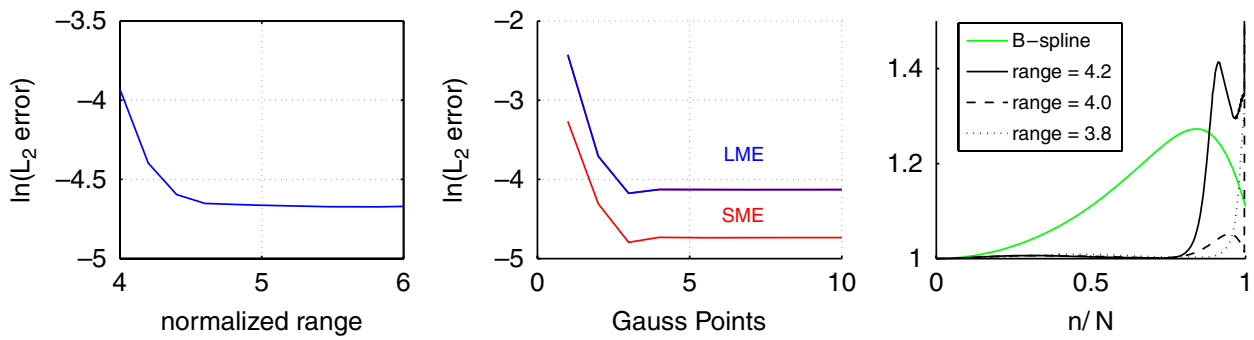

Figure 13. Accuracy depending on evaluation range (left) and number of Gauss points (center) for max-ent approximation, parameter dependence of MLS approximation (right). 
The left plot in Figure 13 shows that again a normalized range of five works well and in the center plot it can be seen that a relatively small number of Gauss points is sufficient for accurate quadrature. The right plot of Figure 13 shows a very important difference between MLS and max-ent approximations. The spectrum results are plotted for the MLS approximants for different choices of the dilation parameter, measuring the support of the shape functions. From the plot it is apparent that the results are very sensitive to this choice. For Figure 12 an optimized parameter is chosen, and as it can be seen in the plot even then the second-order MLS approximation is slightly worse than the linear max-ent approximation and clearly worse than the SME approximation. It should be emphasized that for the SME approximation, no parameter is adjusted in order to optimize results, and a value of $\alpha=4$ is adopted in all the examples presented here. We have also checked that the results are robust with respect to this parameter.

5.1.3. Eigenfrequencies of a membrane. We consider now the $2 \mathrm{D}$ problem of the vibrations of a square membrane, governed by

$$
\Delta u-\omega^{2} u=0 \quad \text { in }(0,1) \times(0,1)
$$

with homogeneous essential boundary conditions in the boundary of the domain, where $\Delta$ is the Laplacian. The exact eigenfrequencies are in this case $\omega_{n m}=\pi \sqrt{m^{2}+n^{2}}$ with $n, m=1,2,3, \ldots$. Figure 14 shows the ratio of numerical to exact frequencies across the spectrum. Again, the plot shows the results for an optimized value of the MLS dilation parameter. It can be observed that in this $2 \mathrm{D}$ example there is a clear hierarchy in the performance of the different methods. SME is the most accurate approach, followed by LME, then quadratic MLS and finally quadratic B-Spline. The right plot emphasizes that slight changes in the dilation parameter of MLS can lead to a drastic degradation of the results. Note also that the optimal dilation parameter for MLS strongly depends on the application, and the optimal value for the beam bending example leads to bad results for the membrane problem.
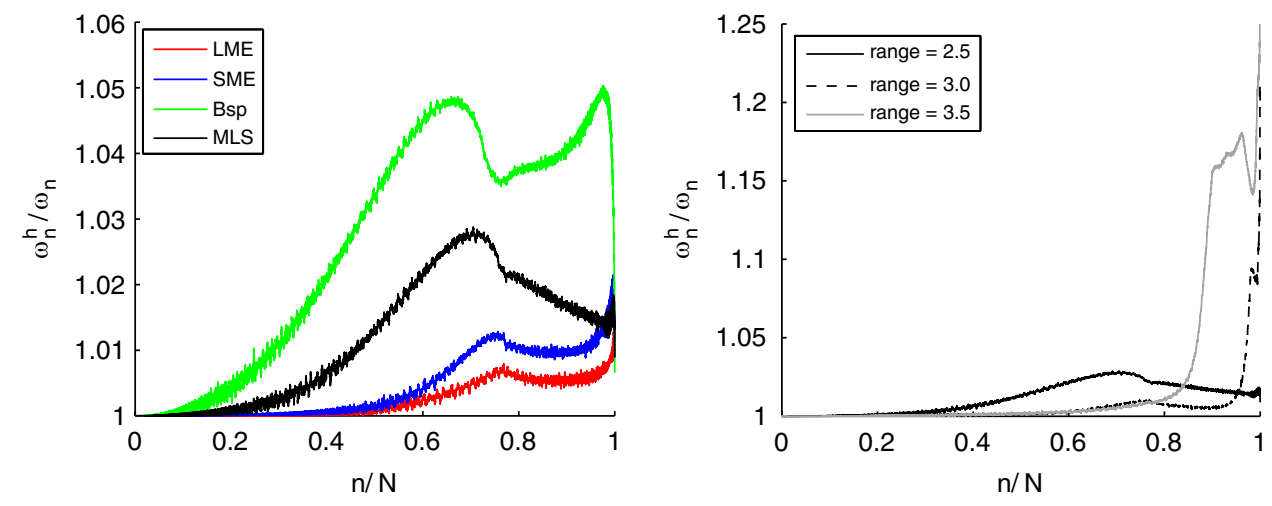

Figure 14. Relative error in the approximation of the eigenfrequency spectrum of a membrane for $99 \times 99$ degrees of freedom with optimized settings for MLS approximation (left plot), comparison of MLS results for different range parameters (right plot). 


\section{J. CYRON, M. ARROYO AND M. ORTIZ}

\subsection{Poisson equation}

We consider now the Poisson equation in $\mathbb{R}^{2}$ and $\mathbb{R}^{3}$ on a unit domain with a source term and boundary conditions so that the exact solution of the problem is known. In $\mathbb{R}^{2}$ we have

$$
\Delta u(x, y)=\sin (2 \pi \omega x) \sin (2 \pi \omega y) \quad \text { for }(x, y) \in \Omega=(0,1) \times(0,1) \text { and }\left.u\right|_{\partial \Omega}=0
$$

where we set $\omega=2$. An approximate solution to (33) is calculated using SME approximants (SME), second-order B-Splines (Bsp), second-order MLS approximants (MLS) and finite elements (Lg2). The MLS shape functions do not satisfy any Kronecker-delta property, and the boundary conditions are enforced by means of the point collocation method [4].

With parameter settings similar to the ones of the above examples the results plotted in Figure 15 are obtained. It is apparent that in $2 \mathrm{D}$ and $3 \mathrm{D}$ optimal convergence is obtained by SME approximants, despite the second-order constraint is violated close to the boundary in a vanishingly small region as the discretization is refined. As a matter of fact, as it can be observed in the convergence plots, in these simulations the convergence rate is slightly better than expected. It is obvious from the figures that while all the presented methods exhibit the same rate of convergence, the accuracy for a given number of DOF greatly depends on the method. The SME approximants provide solutions having orders of magnitude more accurate than finite elements, and considerably more
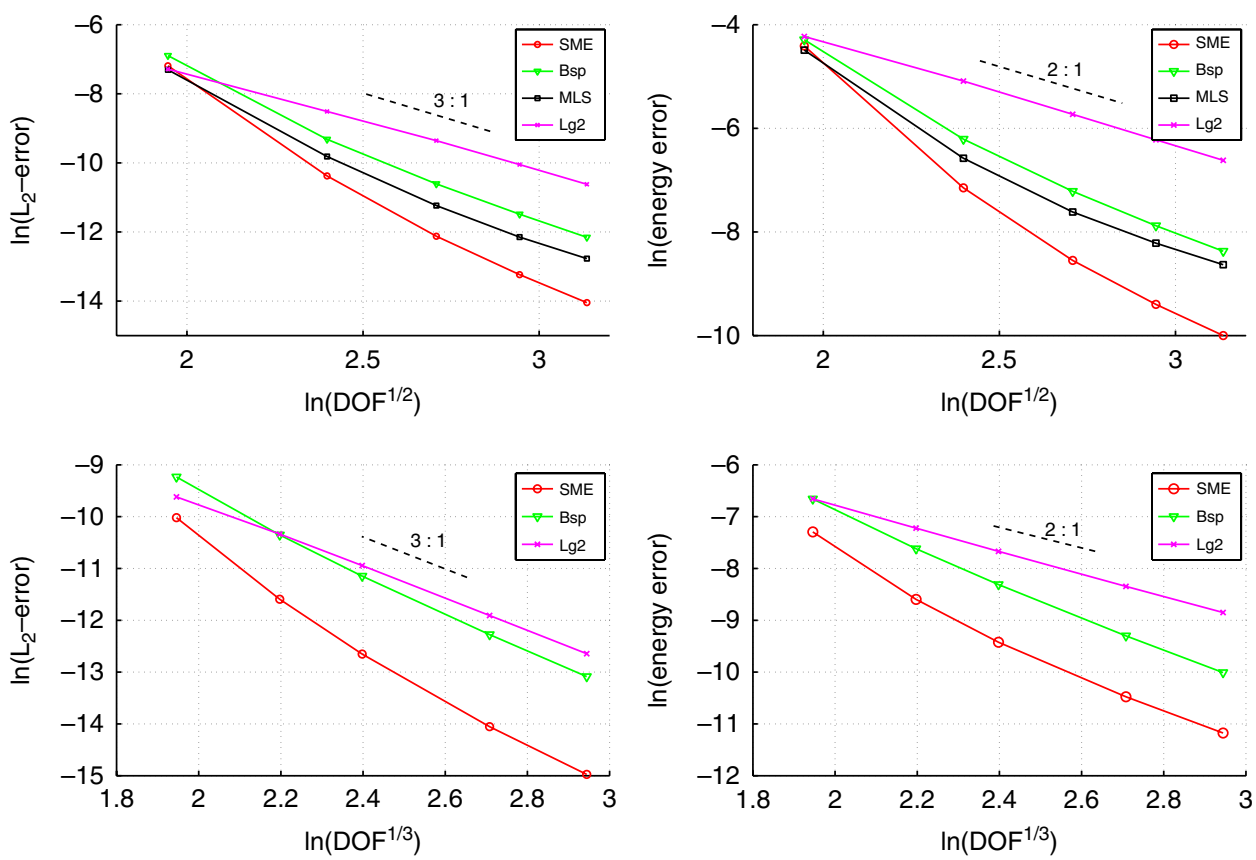

Figure 15. $L_{2}$ error norm (upper left) and energy error (upper right) for the Poisson equation in $\mathbb{R}^{2}, L_{2}$ error norm (lower left) and energy error (lower right) for Poisson equation in $\mathbb{R}^{3}$ with second-order max-ent approximants (SME), second-order B-splines (Bsp), second-order moving-least-squares approximants (MLS) and finite elements (Lg2). 

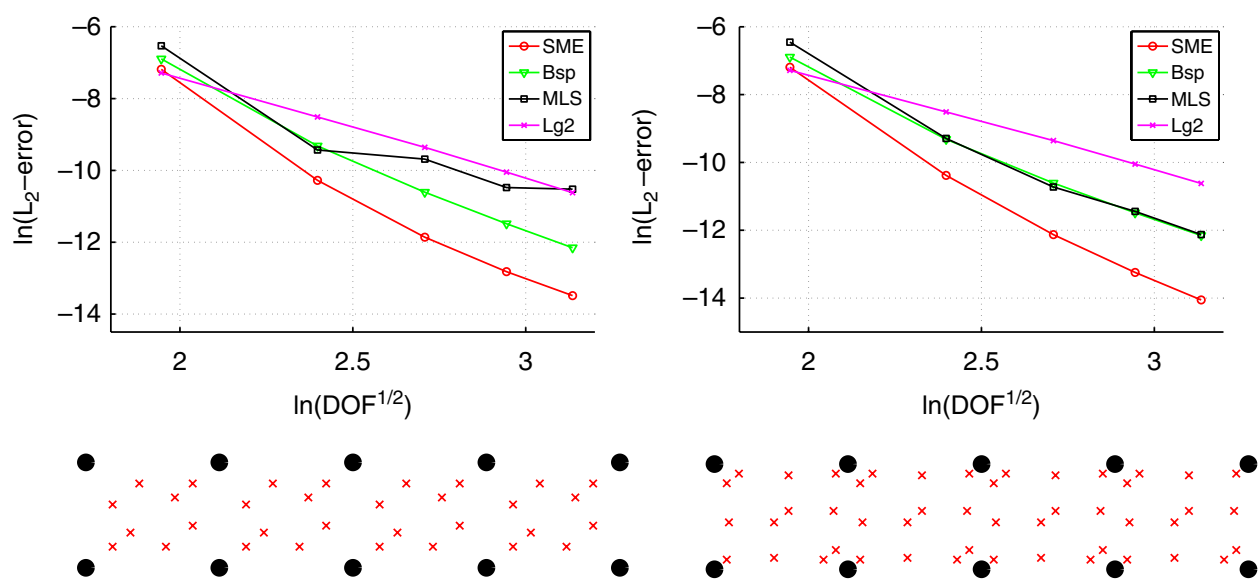

Figure 16. Effect of numerical quadrature on the max-ent and MLS second-order methods. Quadratic finite elements and B-splines are shown for reference, respectively. In the bottom row, the typical density of quadrature points (crosses) relative to the nodes (dots) used to generate the convergence plots are shown.

accurate than B-Splines. MLS provides accurate solutions, but as in the previous examples, the accuracy is found to be very sensitive to the dilation parameter.

Finally, we briefly discuss numerical integration of the meshfree approximation schemes. In the results in Figure 15, quadrature is performed on a triangular background tessellation with a Gauss-Legendre rule with seven points per triangle. Figure 16 shows the effect of using less Gauss points when SME and MLS approximations are used in a Galerkin method. It can be observed that using six points slightly affects the convergence of the MLS method, which still exhibits optimal convergence, while that of SME remains unaffected. When four integration points are used, the quadrature errors dominate the approximation error for the MLS method, whose convergence is destroyed. It can be observed that the optimal convergence of the SME method is maintained. The stronger sensitivity of MLS with regards to quadrature can be understood from Figure 17. The non-negativity of the max-ent approximants makes the corresponding shape functions smoother, without wiggles, in contrast with the MLS shape functions, which display small bumps with negative values. Consequently, the derivatives are much more intricate in the latter case, and the products of these derivatives appearing in the stiffness matrix are in turn more difficult to integrate.

\subsection{Discussion}

We have not attempted a systematic comparison of different methods considered in the present paper accounting for the computational cost and the accuracy. Here, we collect a few observations drawn from our numerical experiments in this respect. Regarding the computational cost, one should distinguish between the cost in the evaluation of the shape functions and its gradients and the cost involved in the numerical solution of a PDE by the Galerkin method, which in turn entails the numerical quadrature and the solution method (eigenvalue problem or solution of a system of equations).

A Galerkin method based on the Lagrange polynomials or B-Spline approximants is clearly the cheapest. Indeed, the evaluation of the shape functions is explicit, hence very fast. The support 

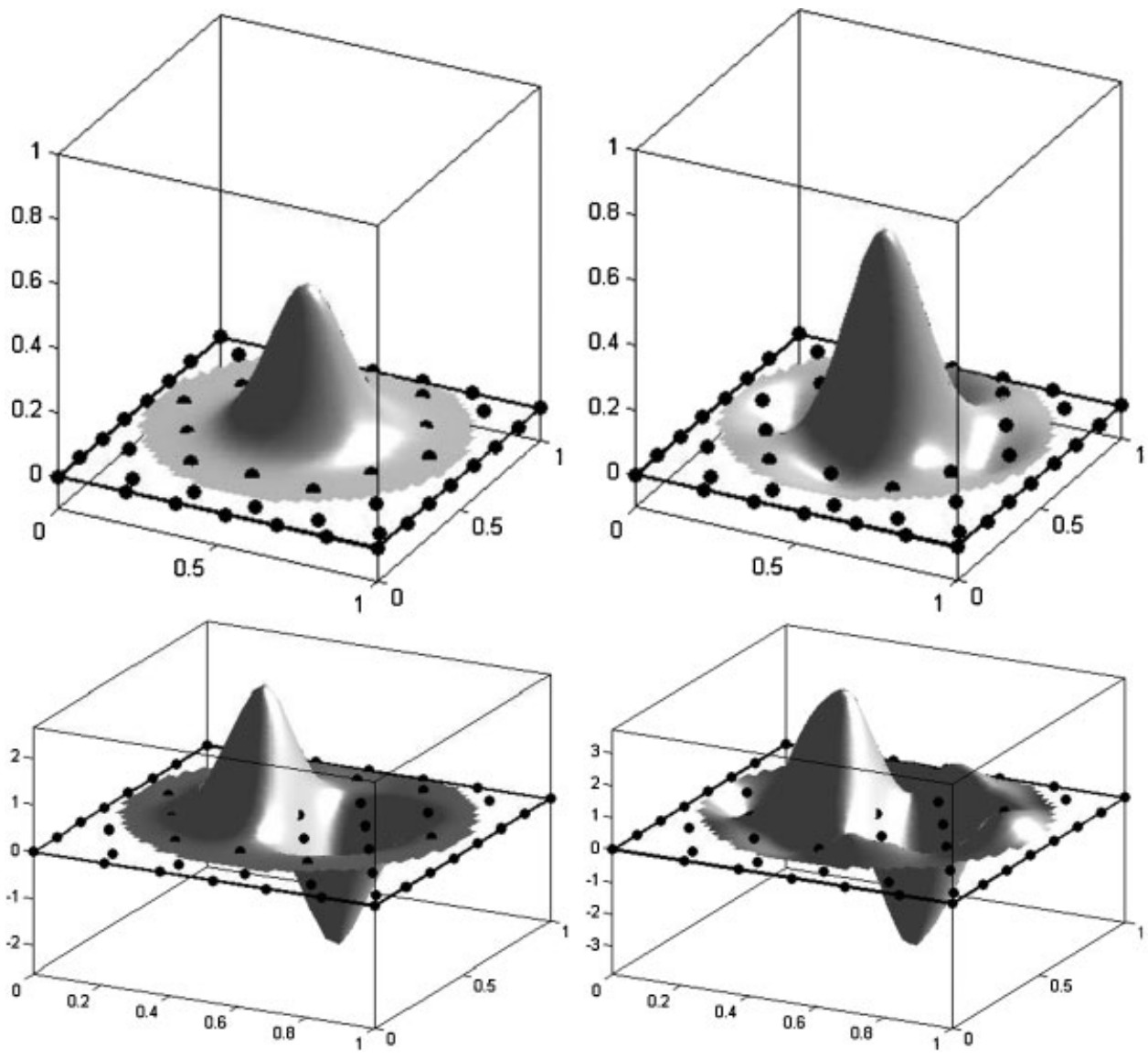

Figure 17. Second-order max-ent and MLS shape functions for a given 2D node set (top) and the derivative with respect to one of the coordinates (bottom).

of the shape functions for a given order is smaller than that of MLS or max-ent approximants, hence leading to smaller bandwidth of the system of equations, and the integration requires fewer quadrature points since polynomials on simple sub-domains need to be integrated. All things considered, in our calculations the over-cost of max-ent calculations with respect to B-Splines is not overwhelming. While comparing max-ent and MLS approximants, our experience indicates that the computational time needed to compute the shape functions and its gradients is typically lower for the max-ent approximants, but we do not want to make a stronger statement since the MATLAB computing times are not a reliable measure of actual computational cost and strongly depend on the programming style. Even considering that the computing cost of the evaluation of these shape functions is equal, the previous section shows that for similar support sizes, the SME approximants require fewer quadrature points than the MLS method.

When accuracy enters the comparison and one considers the computational cost for a given accuracy, the picture is quite different. Indeed, the vibration examples here and elsewhere [7] show that finite elements based on the Lagrange polynomials produce unphysical results in a large portion 
of the spectrum. We have also shown that the MLS method is not robust with respect to the dilation parameter, and that the max-ent approximants provide much more accurate results than a B-Spline method, even of higher order, for a given number of DOF. Taking into account that B-Spline methods do not offer much flexibility in the design of the discretization in multiple dimensions, the numerical experiments suggest that max-ent approximants are a competitive method in vibration analysis application. A similar conclusion is drawn for the Poisson equation, obtaining for a fixed number of DOF much more accurate solutions with the SME method as compared with all other methods studied here.

In trying to extrapolate these conclusions to large-scale possibly non-linear problems, it is important to note that the computational cost involved in the evaluation of the shape functions grows linearly with the number of DOF (for a fixed support size relative to the nodal spacing), whereas the cost of the solution method grows faster for any solution method. Therefore, for large enough problems the cost of the solution method will dominate the overall cost, making max-ent approximants more competitive. For non-linear problems, the relative importance of the cost in the evaluation of the shape functions is much smaller since the shape functions are typically evaluated once at the beginning of the calculation and kept fixed in the iterations or load steps, for instance in a total Lagrangian calculation of finite deformation solid mechanics. This makes max-ent approximants even more attractive. In [1] it was illustrated by 3D non-linear elasticity calculations that LME approximants clearly outperform linear finite elements when computational cost for a given accuracy is considered.

\section{CONCLUSIONS}

We have presented SME approximation schemes, generalizing the first-order schemes presented in [1]. These meshfree approximants are non-negative, $C^{\infty}$, and can be efficiently evaluated in any spacial dimension through duality methods. They possess a weak delta-Kronecker property that makes it trivial to impose boundary values, for instance essential boundary conditions in the Galerkin approximation of PDEs. To our knowledge these approximants are the first second-order non-negative approximants available in arbitrary dimensions and for unstructured data. While in 1D there are many such approximants, for instance B-Splines, and in 2D subdivision approximants or to some degree T-Splines fulfill these requirements, in $\mathbb{R}^{n}$ there are only tensor-product approximants à la NURBS requiring structured data.

The SME approximants are obviously related to other meshfree approximants such as the MLS approximants. They are also close to B-Splines and related technologies since they are nonnegative and smooth. For these reasons, throughout the paper we have emphasized the relations, advantages and drawbacks with respect to these methods. On the basis of numerical experiments of structural vibrations and the numerical solution of the Poisson equation, we have shown that max-ent approximants seem to offer only advantages when compared with MLS approximants: the prescription of boundary data is much simpler, the quadrature is easier, the results are more accurate and they are not plagued by the lack of robustness with respect to the numerical parameters (the dilation parameter) of MLS numerical solutions. As compared with B-Splines and NURBS technologies, the approximants are on one hand certainly more difficult to integrate and do not provide by themselves accurate means of describing complex geometries and on the other hand, they are much more flexible in designing unstructured discretizations in any space dimension, and are smoother. Numerical examples show that they are more accurate than B-Splines (even 


\section{J. CYRON, M. ARROYO AND M. ORTIZ}

higher-order versions, up to quintic B-Splines have been tested), also in structural vibration problems, where B-Splines have been reported to provide excellent results. Numerical experiments suggest that the enhanced accuracy can outweigh the extra computational cost in evaluating the basis functions and in performing numerical quadrature.

Furthermore, the method we present has allowed us to explore the geometry of the consistency conditions for non-negative approximants. While the non-negativity constraint makes it necessary to ask ourselves whether the consistency conditions we impose are feasible, it also endows the approximants with an useful mathematical structure. We have presented a practical method to ensure the feasibility conditions on the basis of heuristic arguments. While this method is robust in practice, it allows for slight violations of the strict second-order reproducing condition. This violation has no consequences on the numerical performance of the approximants. Further mathematical analysis of these issues as well as a better geometric understanding of the moment space in higher dimensions is an avenue for future research.

\section{APPENDIX A: SPACIAL DERIVATIVES OF THE SECOND-ORDER SHAPE FUNCTIONS}

We provide here the spacial derivatives of the SME shape functions. By $s_{a}$ we denote the expression of Equation (22) evaluated for an arbitrary $\lambda$ and $\boldsymbol{\mu}$, i.e. $s_{a}(\mathbf{x}, \boldsymbol{\lambda}, \boldsymbol{\mu})$, and when evaluated at $\lambda^{*}(\mathbf{x})$ and $\boldsymbol{\mu}^{*}(\mathbf{x})$, i.e. for the actual shape functions, we write $s_{a}^{*}$. By $D \lambda^{*}$ we denote the Jacobian matrix of the vector-valued function $\lambda^{*}(\mathbf{x})$, by $D \lambda_{i j}^{*}$ the derivative of the $i$ th element of $\lambda^{*}(\mathbf{x})$ with respect to the $j$ th element of $\mathbf{x}$. The notation for the third-order tensor $D \boldsymbol{\mu}^{*}$ is analogous. The spacial gradient of a function $s_{a}$ is denoted by $\nabla s_{a}$. Furthermore, we denote by $\left(\partial_{\lambda} \log Z\right)^{*}$ the value of $\partial_{\lambda} \log Z$ evaluated for $\lambda=\lambda^{*}$ and $\boldsymbol{\mu}=\boldsymbol{\mu}^{*}$. We also follow the Einstein summation convention.

Before dealing with the actual derivative of the shape functions, we calculate some auxiliary terms. Recall the conditions to determine $\lambda^{*}$ and $\boldsymbol{\mu}^{*}$

$$
\begin{aligned}
& 0=\left(\partial_{\lambda} \log Z\right)^{*}=\sum_{a} s_{a}^{*}\left(\mathbf{x}-\mathbf{x}_{a}\right) \\
& 0=\left(\partial_{\boldsymbol{\mu}} \log Z\right)^{*}=\sum_{a} s_{a}^{*}\left[\left(\mathbf{x}-\mathbf{x}_{a}\right)^{\otimes 2}-\mathbf{G}(\mathbf{x})\right]
\end{aligned}
$$

Since these conditions hold true for every $\mathbf{x}$ throughout the domain, we can differentiate them with respect to $\mathbf{x}$

$$
\begin{array}{r}
\left(\partial_{\lambda \lambda}^{2} \log Z\right)_{i n}^{*} D \lambda_{n k}^{*}+\left(\partial_{\lambda \boldsymbol{\mu}}^{2} \log Z\right)_{i m n}^{*} D \mu_{m n k}^{*}+\left(\partial_{\lambda \mathbf{x}}^{2} \log Z\right)_{i k}^{*}=0 \\
\left(\partial_{\lambda \boldsymbol{\mu}}^{2} \log Z\right)_{n i j}^{*} D \lambda_{n k}^{*}+\left(\partial_{\boldsymbol{\mu \mu}}^{2} \log Z\right)_{i j m n}^{*} D \mu_{m n k}^{*}+\left(\partial_{\boldsymbol{\mu x}}^{2} \log Z\right)_{i j k}^{*}=0
\end{array}
$$

We can conclude from Equations (24) to (28) by means of first- and second-order consistency condition that

$$
\begin{aligned}
& \left(\partial_{\lambda \lambda} \log Z\right)^{*}=\mathbf{G}(\mathbf{x}) \\
& \left(\partial_{\mu \mu} \log Z\right)^{*}=\sum_{a} s_{a}^{*}\left(\mathbf{x}-\mathbf{x}_{a}\right)^{\otimes 4}-\mathbf{G}(\mathbf{x})^{\otimes 2} \\
& \left(\partial_{\lambda \mu} \log Z\right)^{*}=\sum_{a} s_{a}^{*}\left(\mathbf{x}-\mathbf{x}_{a}\right)^{\otimes 3}
\end{aligned}
$$


Furthermore, again by means of first-order consistency condition it is readily verified that

$$
\left(\partial_{\mathbf{x}} s_{a}\right)_{k}^{*}=s_{a}^{*}\left[\mu_{k m}^{*}\left(x-x_{a}\right)_{m}+\mu_{n k}^{*}\left(x-x_{a}\right)_{n}\right]=2 s_{a}^{*} \mu_{k n}^{*}\left(x-x_{a}\right)_{n}
$$

The last step can be done due to the symmetry of $\boldsymbol{\mu}^{*}$. From this equation, together with Equations (24) and (25) it follows that

$$
\begin{aligned}
\left(\partial_{\boldsymbol{\mu x}} \log Z\right)_{i j k}^{*} & =-\left(\partial_{\mathbf{x}} G_{i j}(\mathbf{x})\right)_{k}+2 \sum_{a} s_{a}^{*}\left(x-x_{a}\right)_{i}\left(x-x_{a}\right)_{j}\left(x-x_{a}\right)_{n} \mu_{n k}^{*} \\
\left(\partial_{\lambda \mathbf{x}} \log Z\right)_{i j}^{*} & =\delta_{i j}+\sum_{a}\left(\partial_{\mathbf{x}} s_{a}\right)_{j}^{*}\left(x-x_{a}\right)_{i}=\delta_{i j}+2 \mu_{j n}^{*} G_{n i}(\mathbf{x})
\end{aligned}
$$

Using Equations (A3)-(A7), we see that Equations (A1) and (A2) are a linear system of equations whose unknowns are $D \lambda^{*}$ and $D \boldsymbol{\mu}^{*}$. As already discussed in [1]

$$
\nabla s_{a}^{*}=s_{a}^{*}\left(\nabla f_{a}^{*}-\sum_{b} s_{b}^{*} \nabla f_{b}^{*}\right)
$$

where

$$
f_{a}^{*}=\lambda_{n}^{*}\left(x-x_{a}\right)_{n}+\mu_{m n}^{*}\left[\left(x-x_{a}\right)_{m}\left(x-x_{a}\right)_{n}-G_{m n}(\mathbf{x})\right]
$$

and

$$
\left(\nabla f_{a}^{*}\right)_{k}=\left(\partial_{\mathbf{x}} f_{a}\right)_{k}^{*}+\left(\partial_{\lambda} f_{a}\right)_{n}^{*} D \lambda_{n k}^{*}+\left(\partial_{\boldsymbol{\mu}} f_{a}\right)_{m n}^{*} D \mu_{m n k}^{*}
$$

It is readily verified that

$$
\left(\partial_{\lambda} f_{a}\right)_{n}^{*}=\left(x-x_{a}\right)_{n}
$$

and

$$
\left(\partial_{\boldsymbol{\mu}} f_{a}\right)_{m n}^{*}=\left(x-x_{a}\right)_{m}\left(x-x_{a}\right)_{n}-G_{m n}(\mathbf{x})
$$

We also have

$$
\left(\partial_{\mathbf{x}} f_{a}\right)_{k}^{*}=\lambda_{k}^{*}-\mu_{m n}^{*}\left(\partial_{\mathbf{x}} G_{m n}(\mathbf{x})\right)_{k}+2 \mu_{k n}^{*}\left(x-x_{a}\right)_{n}
$$

With the first- and second-order consistency conditions, we can further simplify the term

$$
\sum_{b} s_{b}^{*}\left(\nabla f_{b}^{*}\right)_{k}=\lambda_{k}^{*}-\mu_{m n}^{*}\left(\partial_{\mathbf{x}} G_{m n}(\mathbf{x})\right)_{k}
$$

Finally, the gradient of the SME shape function $s_{a}^{*}$ can be written as

$$
\left(\nabla s_{a}^{*}\right)_{k}=s_{a}^{*}\left\{\left(x-x_{a}\right)_{n} D \lambda_{k n}^{*}+\left[\left(x-x_{a}\right)_{m}\left(x-x_{a}\right)_{n}-G_{m n}(\mathbf{x})\right] D \mu_{m n k}^{*}+2 \mu_{k n}^{*}\left(x-x_{a}\right)_{n}\right\}
$$

Although these equations seem to be a little involved, they are easy to implement in a computer code. 


\section{J. CYRON, M. ARROYO AND M. ORTIZ}

\section{ACKNOWLEDGEMENTS}

The authors gratefully acknowledge the support of the International Graduate School of Science and Engineering of the Technical University of Munich. M. A. acknowledges the support of the European Commission (Grant No. MIRG-CT-2005-029178), the Ministerio de Ciencia e Innovación (Grant No. DPI2007-61054) and the Generalitat de Catalunya through the prize 'ICREA Academia'. MO gratefully acknowledges the support of the Department of Energy through Caltech's PSAAP Center for the Predictive Simulation of the Dynamic Response of Materials.

\section{REFERENCES}

1. Arroyo M, Ortiz M. Local maximum-entropy approximation schemes: a seamless bridge between finite elements and meshfree methods. International Journal for Numerical Methods in Engineering 2006; 65(13):2167-2202.

2. Belytschko T, Lu Y, Gu L. Element-free Galerkin methods. International Journal for Numerical Methods in Engineering 1994; 37(2):229-256.

3. Liu W, Jun S, Zhang Y. Reproducing kernel particle methods. International Journal for Numerical Methods in Engineering 1995; 20:1081-1106.

4. Huerta A, Belytscko T, Fernández-Méndez S, Rabczuk T. Meshfree Methods. Encyclopedia of Computational Mechanics, vol. 1. Wiley: Chichester, 2004; 279-309.

5. DeVore R. The Approximation of Continuous Functions by Positive Linear Operators. Springer: Berlin, 1972.

6. Hughes T, Cottrell J, Bazilevs Y. Isogeometric analysis: Cad, finite elements, nurbs, exact geometry and mesh refinement. Computer Methods in Applied Mechanics and Engineering 2005; 194:4135-4195.

7. Cottrell J, Reali A, Bazilevs Y, Hughes T. Isogeometric analysis of structural vibrations. Computer Methods in Applied Mechanics and Engineering 2006; 195(41-43):5257-5296.

8. Shannon C. A mathematical theory of communication. The Bell System Technical Journal 1948; 27(3):379-423.

9. Jaynes E. Information theory and statistical mechanics. Physical Review 1957; 106(4):620-630.

10. Arroyo M, Ortiz M. Local maximum-entropy approximation schemes. Meshfree Methods for Partial Differential Equations III. Lecture Notes in Computational Science and Engineering. Springer: Berlin, 2006.

11. Sukumar N, Wright R. Overview and construction of meshfree basis functions: from moving least squares to entropy approximants. International Journal for Numerical Methods in Engineering 2007; 70(2):181-205.

12. Sukumar N. Construction of polygonal interpolants: a maximum entropy approach. International Journal for Numerical Methods in Engineering 2004; 61(12):2159-2181.

13. Prautzch H, Boehm W, Paluszny M. Bézier and B-spline Techniques. Springer: Berlin, 2002.

14. Sukumar N, Moran B, Belytschko T. The natural element method in solid mechanics. International Journal for Numerical Methods in Engineering 1998; 43(5):839-887.

15. Cirak F, Ortiz M, Schröder P. Subdivision surfaces: a new paradigm for thin-shell finite-element analysis. International Journal for Numerical Methods in Engineering 2000; 47(12):2039-2072.

16. Cottin C, Gavrea I, Gonska H, Kacsó D, Zhou D. Global smoothness preservation and variation-diminishing property. Journal of Inequalities and Applications 1999; 4(2):91-114.

17. Boyd S, Vandenberghe L. Convex Optimization. Cambridge University Press: Cambridge, U.K., 2004.

18. Hölling K, Apprich C, Streit A. Introduction to the Web-method and its applications. Advances in Computational Mathematics 2005; 23(1):215-237.

19. Babuška I, Banerjee U, Osborn J. Survey of meshless and generalized finite element methods: a unified approach. Acta Numerica 2003; 12:1-125.

20. Born M, Huang K. Dynamical Theory of Crystal Lattices. Oxford University Press: Oxford, 1954. 